\title{
Gradient-based topology optimization of soft dielectrics as tunable phononic crystals
}

\author{
Atul Kumar Sharma ${ }^{a}$, Majd Kosta ${ }^{c}$, Gal Shmuel ${ }^{b}$, Oded Amir ${ }^{c}$ \\ ${ }^{a}$ Department of Mechanical Engineering, Indian Institute of Technology Jodhpur, Jodhpur 342037, India \\ ${ }^{b}$ Faculty of Mechanical Engineering, Technion-Israel Institute of Technology, Haifa 32000, Israel \\ ${ }^{c}$ Faculty of Civil and Environmental Engineering, Technion-Israel Institute of Technology, Haifa 32000, Israel
}

\begin{abstract}
Dielectric elastomers are active materials that undergo large deformations and change their instantaneous moduli when they are actuated by electric fields. By virtue of these features, composites made of soft dielectrics can filter waves across frequency bands that are electrostatically tunable. To date, to improve the performance of these adaptive phononic crystals, such as the width of these bands at the actuated state, metaheuristics-based topology optimization was used. However, the design freedom offered by this approach is limited because the number of function evaluations increases exponentially with the number of design variables. Here, we go beyond the limitations of this approach, by developing an efficient gradient-based topology optimization method for maximizing the width of the band gaps in an exemplary case study. We employ a finite element formulation of the governing equations, and use the properties of each element as the design variables. In order to iteratively update the design variables, we employ gradient-based optimization, namely the Method of Moving Asymptotes. We carry out and implement fully analytical sensitivity analysis for computing the gradient of the objective function with respect to each one of the design variables. The numerical results of the method developed here demonstrate prohibited frequency bands that are indeed wider that those that were generated using metaheuristics-based topology optimization, while the computational cost to identify them is reduced by orders of magnitude.
\end{abstract}

Keywords: Dielectric elastomers, nonlinear electroelasticity, wave propagation, band gaps, composites, Bloch-Floquet waves, gradient-based topology optimization

\section{Introduction}

Dielectric elastomers (DEs) are active materials that undergo large deformations when they are actuated by electric fields (Pelrine et al., 2000; Hajiesmaili \& Clarke, 2021). Moreover, the constitutive relations of DEs are nonlinear, such that their instantaneous moduli vary as functions of the electromechanical loads. These features have led to the design of various applications, such as haptic devices (Kim et al., 2016), soft robots (Gu et al., 2018), actuators (Hajiesmaili \& Clarke, 2021; Sharma et al., 2018, 2019; Su et al., 2018, 2019; Kashyap et al., 2020; Sharma et al., 2017,

Email addresses: atulksharma@iitj.ac. in (Atul Kumar Sharma), majd-costa@campus.technion.ac.il (Majd Kosta), meshmuel@technion.ac.il (Gal Shmuel), odedamir@technion.ac.il (Oded Amir) 
Ortigosa et al., 2021; Ortigosa \& Martínez-Frutos, 2021; Khurana et al., 2021) and artificial muscles (Lu et al., 2016), that are based on DEs.

More recently, the potential of DEs to function as adaptive waveguides has been explored (Zhu et al., 2020; Mohajer et al., 2021; Jandron \& Henann, 2018; Ziser \& Shmuel, 2017; Wang et al., 2020; Zhu et al., 2020). These studies are based on the understanding that the physical properties that govern elastic waves are electrostatically tunable. Following the pioneering work of Gei et al. (2011) on flexural waves, in a series of papers, Shmuel and collaborators (Shmuel \& Debotton, 2012; Shmuel, 2013; Shmuel \& Pernas-Salomón, 2016; Getz et al., 2017; Getz \& Shmuel, 2017; Bortot \& Shmuel, 2017) have integrated this concept of material tunability with the theory of acoustic band structure, in order to design adaptive wave suppressors. Band theory predicts that waves in elastic composites (also termed phononic crystals) cannot propagate at certain frequencies (band gaps) owing to Bragg reflections, that are functions of the periodicity and the impedance mismatch of the phases (Kushwaha et al., 1993, 1994; Yang \& Kim, 2018; Zhang \& Gao, 2018, Ren et al., 2020). In addition to this wave isolation characteristic in the band gap frequency region, the phononic crystals with local resonators also exhibit the fracture resistance and crack-arrest characteristics which enhance the strength of the structure (Huang et al., 2020, 2021). The idea to the band gaps using DEs is simple: by applying a bias electromechanical field, the periodicity and impedance mismatch between the DE phases is tuned, which in turn change the Bragg reflections and the desired band gaps (Shmuel \& Band, 2016; Lustig \& Shmuel, 2018).

To achieve a significant tunability, aforementioned works have shown that large electric fields are needed-a requirement that hinders the practical utility of DEs as tunable phononic crystals. Since this tunability is a function of the microstructure of the composite, Bortot et al. (2018) have developed a scheme to identify unit cells that exhibit better performance based on topology optimization - a numerical method that systematically searches for optimal material distribution that under specified design and response constraints. As explained in more detail later, here we go beyond this meta-heuristic optimization approach, by developing a gradient-based optimization scheme for the problem considered by Bortot et al. (2018), building upon the model, governing equations and analysis by Shmuel (2013).

Research on topology optimization began with the seminal paper of Bendsøe \& Kikuchi (1988) and recent advancements are discussed in several review articles (Sigmund \& Maute, 2013; Deaton \& Grandhi, 2014). For computational implementation, the design domain-a square unit cell in the current context - is discretized into a finite number of squares and a design variable is attached to each of their centroids. The value of the design variable then indicates which material is placed at the corresponding position in space. This allows for significant design freedom as there is no assumption regarding the topology of the unit cell. Consequently, vast improvement in performance can be achieved in comparison to unit cells of prescribed topology, e.g. a circular fiber within a surrounding matrix.

The use of topology optimization for maximizing phononic band gaps was pioneered by Sigmund \& Søndergaard Jensen (2003). Their work was followed by many studies employing optimization techniques for synthesizing phononic crystals such that desired band gap characteristics are obtained (Halkjær \& Jensen, 2006; Gazonas et al., 2006; Hussein et al., 2007; Bilal \& Hussein, 2011; Liu et al., 2014, 2016a; Li et al., 2016a; Liu et al., 2016b; Xie et al., 2017; Lu et al., 2017; Xie et al., 2017; Chen et al., 2018; Yi et al., 2019; Liu et al., 2020; Quinteros et al., 2021; Zhang et al., 2021). Detailed reviews on topology optimization of phononic crystals were provided by Yi \& Youn (2016) and Li et al. (2019). 
The studies mentioned above aimed at optimizing the linear elastic response, while optimization techniques have been employed only recently for band gaps that depend on nonlinear elastic deformations (Hedayatrasa et al., 2016; Bortot et al., 2018; De Pascalis et al., 2020).

The limitation of these three studies, from the optimal design point of view, is that they are based on metaheuristics, specifically Genetic Algorithms (GA). These methods utilize only function evaluations and no gradient information is used to progress towards an optimum. Their main drawback is that the number of function evaluations that is needed increases exponentially with the number of design variables. Each evaluation consists of solving many generalized eigenvalue problems for generating the band diagram. This computational bottleneck limits the design resolution, i.e., the number of design variables that represent the material distribution, as can be seen in the respective results (Hedayatrasa et al., 2016; Bortot et al., 2018, De Pascalis et al., 2020).

The full potential of topology optimization can only be accessed if gradient-based optimization is used, so that the computational effort can be reduced by orders of magnitude and fine design resolution can be accommodated. Such efficient approach is developed here for the case study analyzed by Shmuel (2013), to which Bortot et al. (2018) applied GA optimization. The medium that they analyzed comprises soft dielectric fibers embedded periodically in a matrix made of a different deformable dielectric. The composite is quasistatically actuated by application of axial electric field. Under the assumption of incompressible phases, Shmuel (2013) has found a closedform solution for the resultant deformation in terms of the bias electric field and the properties of the composite. Using a plane-wave expansion approach, Shmuel (2013) determined the band structure of incremental anti-plane waves propagating in the deformed configuration, the gaps of which were optimized by Bortot et al. (2018) using a GA method.

Here, we introduce a finite element formulation of the incremental problem, and use the properties of each element as the design variables, the objective of which is to maximize the width of the gap in the audible frequency range. In order to iteratively update the design variables, we employ gradientbased optimization, namely the Method of Moving Asymptotes-MMA (Svanberg, 1987). We carry out and implement fully analytical sensitivity analysis for computing the gradient of the objective function with respect to each one of the design variables. We generate optimized designs with superior performance compared to those obtained by Bortot et al.(2018), while the computational cost - in terms of the number of function evaluations - is smaller by orders of magnitude.

Before we proceed with details on the structure of this paper, it is important to note that the constitutive model upon which we develop our equations neglects viscosity (Destrade \& Saccomandi, 2004; Hong, 2011; Chiang Foo et al., 2012), to establish a starting point for gradient-based optimization in a simpler setting. While our assumption is reasonable for silicons, it is not appropriate for acrylics (Kornbluh \& Pelrine, 2008), there viscosity is much more pronounced, and future work will require the integration of viscoelastic theory into (see, e.g., the way in which Mohajer et al. (2021) extended the work of Shmuel et al. (2012)). We also emphasize that the dynamic problem considered here focuses exclusively on the anti-plane motion, i.e., assumes that the more complicated in-plane motion is not excited. This assumption significantly simplifies the analysis; a more complete investigation, which allows motions, is left for future work, and would require the framework of Getz et al. (2017).

The paper is organized as follows. In Sec. 2 we provide the governing equations of electroelastic composites (Dorfmann \& Ogden, 2005; Suo et al., 2008; Gei et al., 2013; DeBotton et al., 2007; Dorfmann \& Ogden, 2010), upon which the forthcoming analysis is based. In Sec. 3 we recall the solution of Shmuel (2013) to the quasistatic deformation of the periodic DE composite owing to 
axial electric fields, and the equations developed therein for the incremental problem of anti-plane shear waves. In Sec. 4, we present the finite element formulation for the solution of the incremental problem, and specifically for the calculation of the band diagram of the deformed DE composite. The contribution of this work is detailed in Sec. 5 and Sec. 6, where we present the analytical design sensitivity analysis for computing the gradient of the objective function with respect to each one of the design variables; implement the method using in-house MatLab code; and present numerical examples. We conclude this paper with a summary of our results in Sec.7.

\section{Governing equations}

To provide a self-contained report, here we briefly summarize the governing equations pertaining to nonlinear electro-mechanical deformation of dielectric elastomers following the theory of nonlinear electroelasticity (Dorfmann \& Ogden, 2005; Suo et al., 2008) and the associated linearized incremental theory (Dorfmann \& Ogden, 2010).

\subsection{Nonlinear electroelasticity theory}

Consider an arbitrary deformable dielectric composite body $\mathcal{B}^{m} \cup \mathcal{B}^{f}=\mathcal{B}$ made up of two incompressible homogeneous dielectric phases $\mathcal{B}^{m}$ and $\mathcal{B}^{f}$, and surrounded by vacuum. In the undeformed configuration, the dielectric composite occupies a region $\mathcal{B}_{0}^{m} \cup \mathcal{B}_{0}^{f}=\mathcal{B}_{0}$ with the boundary $\partial \mathcal{B}_{0}$. An arbitrary material particle in this configuration is denoted by its position vector $\mathbf{X}$. When subjected to electromechanical loadings, the dielectric composite body is deformed and occupies a region $\mathcal{B}_{t}^{m} \cup \mathcal{B}_{t}^{f}=\mathcal{B}_{t}$ with boundary $\partial \mathcal{B}_{t}$. The position of a material particle in the deformed configuration is denoted by $\mathbf{x}$, which is related to $\mathbf{X}$ through the nonlinear deformation map $\mathbf{x}=\chi(\mathbf{X}, t)$. The components of deformation gradient are given by $F_{i J}=\frac{\partial \chi_{i}(\mathbf{X}, t)}{\partial X_{J}}$. The determinant of the deformation gradient relates the volume ratio of an infinitesimal element in the deformed state, $d v$, and its counterpart in the undeformed state, $d V$, such that $J=\operatorname{det}(\mathbf{F})>0$. The components of the right Cauchy-green tensor are $C_{I J}=F_{i I} F_{i J}$.

For quasi-electrostatic processes, in the absence of free charges and currents, Gauss's law and Faraday's law are written as

$$
\frac{\partial d_{i}}{\partial x_{i}}=0, \quad \frac{\partial e_{j}}{\partial x_{k}} \epsilon_{i j k}=0_{i},
$$

respectively, where $\mathbf{d}$ and $\mathbf{e}$ are the electric displacement and the electric field vectors in the deformed configuration, respectively; 0 represents the zero vector and $\epsilon$ represents the Levi-Civita symbol. In addition, the mechanical equilibrium equation in the deformed configuration, in the absence of mechanical body forces, is

$$
\frac{\partial \sigma_{i j}}{\partial x_{j}}=0_{i}
$$

where $\boldsymbol{\sigma}$ is the symmetric total Cauchy stress tensor consisting of both mechanical and electrical contributions.

In this paper, we consider infinite, periodic, dielectric composites, which posses internal, mechanical traction and free charge interfaces between the two dielectric phases $m$ and $f$ with jump boundary conditions

$$
\llbracket \sigma_{i j} \rrbracket n_{j}=0_{i}, \quad \llbracket d_{i} \rrbracket n_{i}=0, \quad \llbracket e_{i} \rrbracket n_{j} \epsilon_{i j k}=0_{k}
$$


where $\llbracket \bullet \rrbracket=(\bullet)^{m}-(\bullet)^{f}$ represents the jump of quantities between the two phases, and $\mathbf{n}$ represents the unit normal vector on the deformed interface and directed from phase $m$ to phase $f$.

In Lagrangian description that uses $\mathbf{X}$ as the independent variable, the total first Piola-Kirchhoff stress tensor is related to the total Cauchy stress tensor by $P_{i J}=J \sigma_{i k} F_{k J}^{-\mathrm{T}}$ and satisfies the Lagrangian form of the equilibrium equation

$$
\frac{\partial P_{i J}}{\partial X_{J}}=0_{i}
$$

in the undeformed configuration $\mathcal{B}_{0}$. The Lagrangian electric displacement and electric field are given as $D_{J}=J F_{J i}^{-1} d_{i}$ and $E_{J}=F_{i J} e_{i}$, respectively. They satisfy the Lagrangian form of Gauss's law and Faraday's law

$$
\frac{\partial D_{J}}{\partial X_{J}}=0, \quad \frac{\partial E_{J}}{\partial X_{K}} \epsilon_{I J K}=0_{I}
$$

respectively.

We consider non-dissipative materials for which the first Piola-Kirchoff stress tensor $\mathbf{P}$ and Lagrangian electric field $\mathbf{E}$ are obtained constitutively in terms of the deformation gradient $\mathbf{F}$ and Lagrangian electric displacement $\mathbf{D}$ through an augmented energy density function $\psi(\mathbf{F}, \mathbf{D})$ as (Dorfmann \& Ogden, 2005)

$$
P_{i J}=\frac{\partial \psi}{\partial F_{i J}}-p_{0} F_{i J}^{-\mathrm{T}}, \quad E_{J}=\frac{\partial \psi}{\partial D_{J}},
$$

where $p_{0}$ is a Lagrange multiplier that accounts for the incompressibility constraint.

\subsection{The linearized incremental theory}

Following Dorfmann \& Ogden (2010), we present the governing equations pertaining to the time dependent infinitesimal increment in both elastic and electric displacement fields $\dot{\mathbf{x}}=\dot{\chi}(\mathbf{X}, t)$, $\dot{\mathbf{D}}(\mathbf{X}, t)$, respectively, superimposed on the static deformed configuration characterized by the fields $\chi(\mathbf{X}, t)$ and $\mathbf{D}(\mathbf{X}, t)$. Henceforth, the infinitesimal incremental quantities are denoted with the superposed dot. The push-forwards of increments in the total first Piola-Kirchhoff stress, the Lagrangian electric displacement and the Lagrangian electric fields are

$$
\Sigma_{i j}=\frac{1}{J} \dot{P}_{i K} F_{j K}, \quad \check{d}_{i}=\frac{1}{J} F_{i J} \dot{D}_{J}, \quad \check{e}_{i}=F_{J i}^{-1} \dot{E}_{J}
$$

respectively, satisfying the incremental forms of Gauss's, Faraday's laws, and momentum balance equation,

$$
\frac{\partial \check{d}_{i}}{\partial x_{i}}=0, \quad \frac{\partial \check{e}_{j}}{\partial x_{k}} \epsilon_{i j k}=0_{i}, \quad \frac{\partial \Sigma_{i j}}{\partial x_{j}}=\rho \frac{\partial^{2} \dot{x}_{i}}{\partial t^{2}},
$$

respectively, where $\dot{\mathbf{x}}(\mathbf{x}, t):=\dot{\chi}(\mathbf{X}, t)$.

For an incompressible dielectric elastomer material, the linearized incremental constitutive laws in terms of the increments $\Sigma$ and ě are obtained using Eq. (6) and the push-forward operation as

$$
\begin{aligned}
& \Sigma_{i j}=\mathscr{C}_{i j k l} h_{k l}+p_{0} h_{j i}-\dot{p}_{0} \delta_{i j}+\mathscr{B}_{i j k} \check{d}_{k}, \\
& \check{e}_{i}=\mathscr{B}_{l k i} \dot{h}_{k l}+\mathscr{A}_{i j} \check{d}_{j},
\end{aligned}
$$




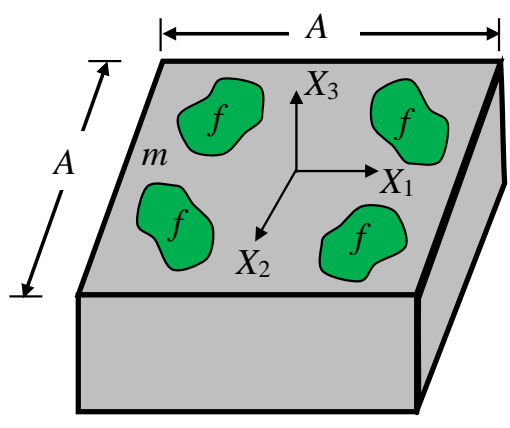

(a)

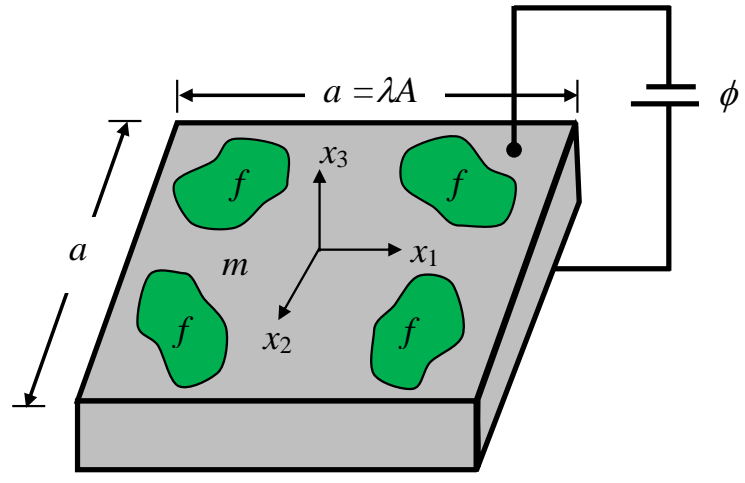

(b)

Figure 1: Dielectric elastomer fiber composite in the (a) undeformed configuration, and (b) deformed configuration, when subjected to an axial electric field.

where

$$
\begin{aligned}
& \mathscr{C}_{i j k l}=\frac{1}{J} F_{j I} \frac{\partial^{2} \psi}{\partial F_{i I} \partial F_{k J}} F_{l J}, \quad \mathscr{B}_{i j k}=F_{j I} \frac{\partial^{2} \psi}{\partial F_{i I} \partial D_{J}} F_{J k}^{-1} \\
& \mathscr{A}_{i j}=J F_{I i}^{-1} \frac{\partial^{2} \psi}{\partial D_{I} \partial D_{J}} F_{J j}^{-1}
\end{aligned}
$$

are the spatial constitutive tangent moduli, and $h_{i j}=\frac{\partial \dot{x}_{i}}{\partial x_{j}}$ is subjected to incompressibility constraint $h_{k k}=0$.

\section{Quasistatic finite deformation and incremental anti-plane shear waves in DE composites}

As mentioned, using the above theory, Shmuel (2013) determined the quasistatic deformation of the periodic DE composite owing to axial electric fields [see also the study by Sharma et al. (2021)], and developed the equations that govern incremental anti-plane shear waves propagating through the deformed composite. In order to provide a self-contained report, we concisely recapitulate the quasistatic solution and incremental equations, to which we will employ a finite element solution and develop the optimization process.

We consider a dielectric elastomer composite that is infinitely periodic in the $\left(x_{1}, x_{2}\right)$ plane. The composite is made of incompressible DE fibers along the $x_{3}$ direction with arbitrary cross-section (phase $f$ ), that are embedded in a different incompressible DE matrix (phase $m$ ). The constitutive response of the phases is given by the augmented Gentian free energy density function (Zhao et al. 2007; Sharma, 2020):

$$
\psi\left(F_{i J}, D_{P}\right)=-\frac{\mu J_{m}}{2} \ln \left[1-\frac{F_{i J} F_{i J}-3}{J_{m}}\right]+\frac{1}{2 \varepsilon} D_{P} F_{i P} F_{i Q} D_{Q}
$$

where $\varepsilon$ is the dielectric permittivity, $\mu$ is the shear modulus of the dielectric elastomer, $J_{m}$ is a dimensionless material parameter that accounts for the strain stiffening that arises from the limited extensibility of the elastomers. Figure 1 a shows the schematic of a square DE composite unit cell 
of length $A$ in the undeformed configuration. The composite is subjected to an average electric field of magnitude $e_{3}$ in the $x_{3}$ direction, and is free to expand in the transverse $\left(x_{1}, x_{2}\right)$ plane. From an experimental viewpoint, such actuation is achieved by the application of a voltage drop between two compliant coated electrodes on the top and bottom faces of the composite, located far enough one of each other to avoid edge effects. Shmuel (2013) has shown that the resultant deformation and electric field are homogeneous, in the form

$$
\mathbf{F}^{(m)}=\mathbf{F}^{(f)}=\left[\begin{array}{ccc}
\lambda & 0 & 0 \\
0 & \lambda & 0 \\
0 & 0 & \lambda^{-2}
\end{array}\right], \quad \mathbf{e}^{(m)}=\mathbf{e}^{(f)}=\left[\begin{array}{c}
0 \\
0 \\
e_{3}
\end{array}\right],
$$

where the in-plane stretch ratio $\lambda$ is given by the solution of the nonlinear equation

$$
\overline{\check{\mu}}\left(\lambda^{2}-\lambda^{-4}\right)=\bar{\varepsilon} e_{3}^{2},
$$

where $\check{\mu}^{(p)}=\frac{\mu^{(p)} J_{m}^{(p)}}{J_{m}^{(p)}-2 \lambda^{2}-\lambda^{-4}+3},(\bar{\bullet})=v^{(m)}(\bullet)+v^{(f)}(\bullet)$ and $v^{(p)}$ is the volume fraction of phase $p$. Thus, up to the point of bifurcation, for any given value of applied electric field $e_{3}$ and properties of the composite, the solution of Eq. (13) delivers the resultant deformation.

On the top of deformed configuration, we consider anti-plane waves propagating in the $\left(x_{1}, x_{2}\right)$ plane of the composite. The size of the composite in this plane is assumed large enough to be analyzed as an infinite periodic medium. Figure 2 a shows the schematic of the DE fiber composite unit cell in the deformed $\left(x_{1}, x_{2}\right)$ plane. Let $\dot{x}_{3}(\mathbf{x}, t)$ and $\dot{\phi}(\mathbf{x}, t)$ denote the anti-plane incremental displacement and the incremental electric potential, respectively; the equations that govern these two unknown fields are

$$
\begin{gathered}
\frac{\partial}{\partial x_{j}}\left(\tilde{\mu}(\mathbf{x}) \frac{\partial \dot{x}_{3}(\mathbf{x}, t)}{\partial x_{j}}-\tilde{d}(\mathbf{x}) \frac{\partial \dot{\phi}(\mathbf{x}, t)}{\partial x_{j}}\right)=\rho(\mathbf{x}) \frac{\partial^{2} \dot{x}_{3}}{\partial t^{2}}, \\
\frac{\partial}{\partial x_{j}}\left(-\tilde{d}(\mathbf{x}) \frac{\partial \dot{x}_{3}(\mathbf{x}, t)}{\partial x_{j}}-\varepsilon(\mathbf{x}) \frac{\partial \dot{\phi}(\mathbf{x}, t)}{\partial x_{j}}\right)=0
\end{gathered}
$$

respectively, where $j=1,2, \tilde{\mu}(\mathbf{x})=\check{\mu}(\mathbf{x}) \lambda^{2}-\varepsilon(\mathbf{x}) e_{3}^{2}$, and $\tilde{d}(\mathbf{x})=\varepsilon(\mathbf{x}) e_{3}$.

Eqs. (14)-(15) are the starting point of our analysis: in the next Sec. we develop a finite element formulation to solve them, and in turn obtain the band diagram of incremental anti-plane shear waves in the DE composite. Later on, based on the finite element formulation, we will develop a gradient-based topology optimization method, the objective of which is to identify optimal unitcells that generate the widest band gaps at designated electric fields.

\section{Finite element formulation}

We introduce next a finite element formulation with Bloch-Floquet periodic boundary conditions (Kittel et al., 1996) to solve Eqs. (14)-(15). With $\delta \dot{x}_{3}$ and $\delta \dot{\phi}$ denoting virtual incremental anti-plane displacement and virtual incremental electric potential, respectively, the weak form statements 

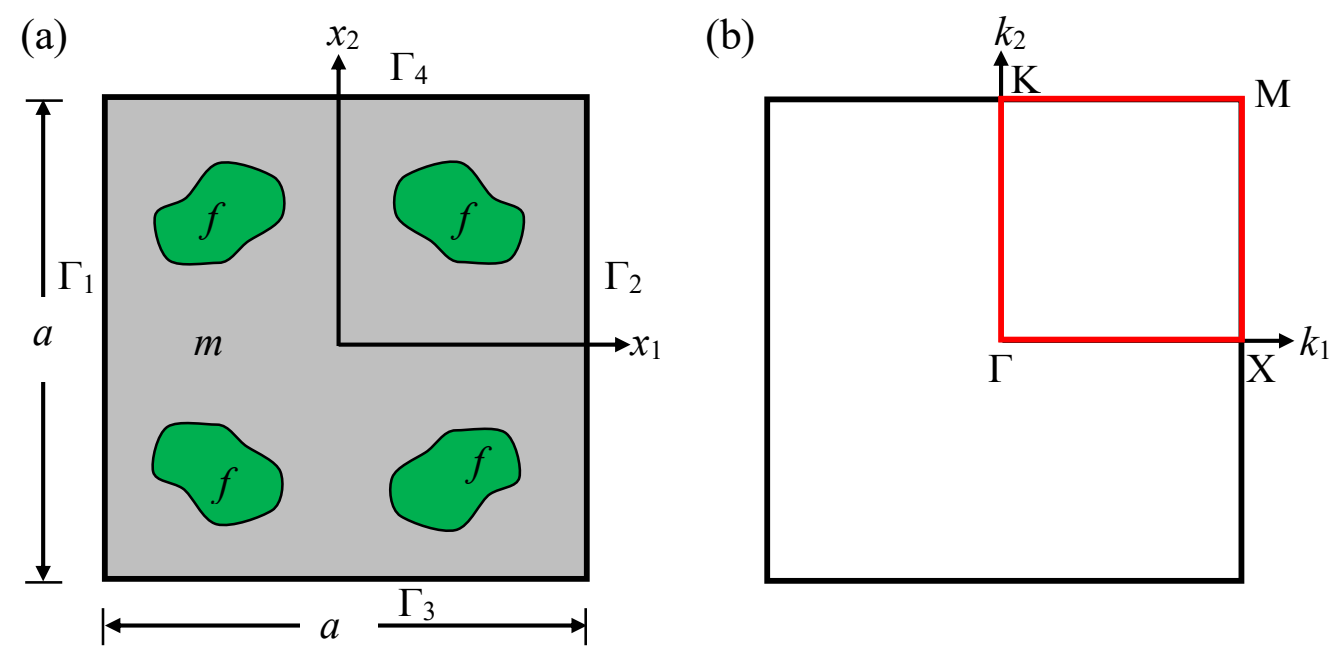

Figure 2: (a) Schematic description of the twice-symmetric square unit cell in the periodicity plane $\left(x_{1}, x_{2}\right)$, and (b) the corresponding first Brillouin zone with line segments to be scanned for determining the gaps extrema.

corresponding to Eq. (14) and Eq. (15) are written as

$$
\begin{gathered}
\int_{\mathcal{B}_{t}}\left(\tilde{\mu} \frac{\partial \dot{x}_{3}}{\partial x_{j}}-\tilde{d} \frac{\partial \dot{\phi}}{\partial x_{j}}\right) \frac{\partial \delta \dot{x}_{3}}{\partial x_{j}} d s=\int_{\mathcal{B}_{t}} \rho \delta \dot{x}_{3} \frac{\partial^{2} \dot{x}_{3}}{\partial t^{2}} d s, \\
\int_{\mathcal{B}_{t}}\left(-\tilde{d} \frac{\partial \dot{x}_{3}}{\partial x_{j}}-\varepsilon \frac{\partial \dot{\phi}}{\partial x_{j}}\right) \frac{\partial \delta \dot{\phi}}{\partial x_{j}} d s=0,
\end{gathered}
$$

respectively, where $s$ is the area of the unit cell in the deformed configuration.

The DE composite unit cell is discretized using finite elements, $\mathcal{B}_{t}=\cup \mathcal{B}_{t}^{e}$, and finite element approximation for the incremental displacement and electric potential fields inside the element $\mathcal{B}_{t}^{e}$ are written using the Voigt notation as

$$
\dot{x}_{3}=N_{a} \dot{x}_{a 3} ; \dot{\phi}=N_{a} \dot{\phi}_{a},
$$

where $N_{a}$ represents the shape function associated with node $a$ of the element, and $\dot{x}_{a 3}$ and $\dot{\phi}_{a}$ are the incremental anti-plane displacement and the incremental electric potential values at node a. Substituting above finite approximations and the linearized constitutive relations of Eq. (9) into Eqs. (16)-(17), the elemental level equations corresponding to momentum balance and Gauss law are written as

$$
\begin{gathered}
{\left[K^{m m}\right]\left\{\dot{x}_{b 3}\right\}+\left[K^{m e}\right]\left\{\dot{\phi}_{b}\right\}=[M]\left\{\frac{\partial^{2} \dot{x}_{b 3}}{\partial t^{2}}\right\},} \\
{\left[K^{e m}\right]\left\{\dot{x}_{b 3}\right\}+\left[K^{e e}\right]\left\{\dot{\phi}_{b}\right\}=0,}
\end{gathered}
$$

where $K^{m m}$ is the mechanical stiffness matrix, $K^{m e}$ and $K^{e m}$ are the electro-mechanical coupled 
stiffness matrices, $K^{e e}$ is the electric stiffness matrix, and $M$ is the mass matrix. The expressions for these elemental stiffness matrices are given as

$$
\begin{aligned}
K^{m m} & =\int_{\mathcal{B}_{t}^{e}} \tilde{\mu} \frac{\partial N_{a}}{\partial x_{j}} \frac{\partial N_{b}}{\partial x_{j}} d s, \\
K^{m e} & =-\int_{\mathcal{B}_{t}^{e}} \tilde{d} \frac{\partial N_{a}}{\partial x_{j}} \frac{\partial N_{b}}{\partial x_{j}} d s, \\
K^{e m} & =-\int_{\mathcal{B}_{t}^{e}} \tilde{d} \frac{\partial N_{a}}{\partial x_{j}} \frac{\partial N_{b}}{\partial x_{j}} d s, \\
K^{e e} & =-\int_{\mathcal{B}_{t}^{e}} \varepsilon \frac{\partial N_{a}}{\partial x_{j}} \frac{\partial N_{b}}{\partial x_{j}} d s, \\
M & =\int_{\mathcal{B}_{t}^{e}} \rho N_{a} N_{b} d s .
\end{aligned}
$$

Further, by assuming both the nodal incremental displacement and the incremental electric potential to be time harmonic as

$$
\dot{x}_{b 3}=\tilde{x}_{b}(x) e^{-i \omega t} ; \quad \dot{\phi}_{b}=\tilde{\phi}_{b}(x) e^{-i \omega t},
$$

and inserting these harmonic expressions into Eqs. (19)-(20), we get the incremental elemental level equations governing the electro-elastic wave motion with angular frequency $\omega$ as

$$
\begin{gathered}
{\left[K^{m m}\right]\left\{\tilde{x}_{b}\right\}+\left[K^{m e}\right]\left\{\tilde{\phi}_{b}\right\}-\omega^{2}[M]\left\{\tilde{x}_{b}\right\}=0,} \\
{\left[K^{e m}\right]\left\{\tilde{x}_{b}\right\}+\left[K^{e e}\right]\left\{\tilde{\phi}_{b}\right\}=0,}
\end{gathered}
$$

where $\tilde{x}_{b}$ and $\tilde{\phi}_{b}$ denote the spatially dependent nodal incremental displacement and electric potential fields, respectively. Eliminating $\tilde{\phi}_{b}$ from Eq. 27] by substituting $\left\{\tilde{\phi}_{b}\right\}=-[G]\left[K^{e m}\right]\left\{\tilde{x}_{b}\right\}$ from Eq. (28), we finally obtain the following eigenvalue problem

$$
\begin{aligned}
& \left(\left[K^{m m}-K^{m e} G K^{e m}\right]-\omega^{2}[M]\right)\left\{\tilde{x}_{b}\right\} \\
& =\left(\left[K^{*}\right]-\omega^{2}[M]\right)\left\{\tilde{x}_{b}\right\}=0,
\end{aligned}
$$

where $K^{*}=K^{m m}-K^{m e} G K^{e m}$ and $G$ denotes the Moore-Penrose pseudoinverse of the electric stiffness matrix $K^{e e}$

Using the Bloch theorem (Kittel et al., 1996), we specify the periodic boundary conditions for analyzing the anti-plane wave propagation through the infinite, periodic DE composite. For the DE composite with square lattice shown in Fig. 2a, the Bloch periodic boundary conditions for the 
nodal displacements are expressed as (Vatanabe et al., 2014; Jandron \& Henann, 2018)

$$
\begin{aligned}
& \tilde{x}_{b}\left(x_{\Gamma_{2}}\right)=\exp \left(i k_{1} a\right) \tilde{x}_{b}\left(x_{\Gamma_{1}}\right), \\
& \tilde{x}_{b}\left(x_{\Gamma_{4}}\right)=\exp \left(i k_{2} a\right) \tilde{x}_{b}\left(x_{\Gamma_{3}}\right) .
\end{aligned}
$$

Similarly, the Bloch periodic boundary conditions for the nodal electric potential fields are expressed as

$$
\begin{aligned}
& \tilde{\phi}_{b}\left(x_{\Gamma_{2}}\right)=\exp \left(i k_{1} a\right) \tilde{\phi}_{b}\left(x_{\Gamma_{1}}\right), \\
& \tilde{\phi}_{b}\left(x_{\Gamma_{4}}\right)=\exp \left(i k_{2} a\right) \tilde{\phi}_{b}\left(x_{\Gamma_{3}}\right),
\end{aligned}
$$

where $k_{1}$ and $k_{2}$ are the component of real Bloch wave vector $\mathbf{k}=k_{i} e_{i}$ along $x_{1}$ and $x_{2}$ directions, as shown in Fig. 2p. In order to implement these complex-valued boundary conditions, we used the augmented penalty method (Felippa, 2001; Oliveira et al., 2010).

The eigenvalue problem stated in Eq. (29) along with the Bloch periodic boundary conditions of Eqs. (30)-(31) is solved for computing the band diagram, i.e, $\omega$ as a function of the wave vector k. Due to the quarter symmetry of the problem considered in this paper (see Fig. 2a), it is sufficient to consider only the wave vector along the edges $[\Gamma-\mathrm{X}-\mathrm{M}-\mathrm{K}-\Gamma]$ of the first Brillouin zone, marked by red lines in Fig. 2b (Sigmund \& Søndergaard Jensen, 2003; Vatanabe et al., 2014; Meng et al., 2017; Yi \& Youn, 2016). The coordinates of various points along these edges are as $\Gamma=(0,0), \mathrm{X}=(\pi / a, 0), \mathrm{M}=(\pi / a, \pi / a)$, and $\mathrm{K}=(0, \pi / a)$.

Having at hand a finite-element formulation to calculate the band diagram, we proceed next to develop the gradient-based topology optimization method, whose aim is to find the microstructure that results with the widest band gaps at prescribed electric fields.

\section{Topology optimization}

In the design of phononic band gap structures, we need to find the unit cell that yields the desired band gap characteristics. In this section, we introduce the topology optimization method for obtaining the optimized distribution of the matrix and fiber phases in the DE composite unit cell that maximizes the band gap size.

\subsection{Material property interpolation for topology optimization}

In this work, the design domain is a unit cell that is discretized by finite elements. To interpolate the material properties for each finite element used to discretize the design domain, artificial design variables $\xi^{e} \in[0,1](e=1,2, \ldots, N, N$ being the total number of finite elements in the design domain) are assigned to all finite elements as shown in Fig. 3 .

For example, $\xi^{e}=0$ represents an element $e$ that is composed of matrix phase, while $\xi^{e}=1$ represents an element consisting of fiber phase. As discussed by Sigmund \& Søndergaard Jensen (2003) and Meng et al. (2017), the material properties for each finite element in the design domain can be interpolated linearly between the matrix and fiber phases as

$$
\begin{gathered}
\rho^{e}=\left(1-\xi^{e}\right) \rho^{(m)}+\xi^{e} \rho^{(f)}, \\
\mu^{e}=\left(1-\xi^{e}\right) \mu^{(m)}+\xi^{e} \mu^{(f)},
\end{gathered}
$$




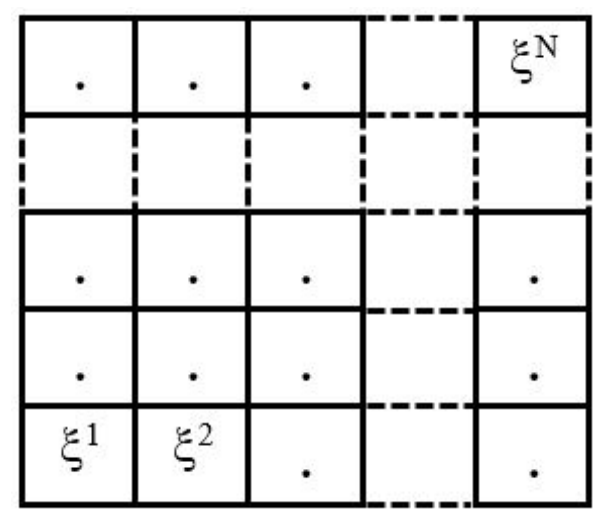

Figure 3: Discretization of the unit cell into $N$ finite elements and assignment of a design variable to each finite element.

$$
\begin{gathered}
\varepsilon^{e}=\left(1-\xi^{e}\right) \varepsilon^{(m)}+\xi^{e} \varepsilon^{(f)} \\
J_{m}^{e}=\left(1-\xi^{e}\right) J_{m}^{(m)}+\xi^{e} J_{m}^{(f)} .
\end{gathered}
$$

\subsection{Optimization problem}

The topology optimization problem aims to maximize the band gap width between the two adjacent bands in the audible frequency range 0-10 kHz. For two adjacent bands, assuming the eigenfrequencies corresponding to overlying band and underlying band to be $\omega_{j+1}$ and $\omega_{j}$, respectively, the objective function may be written as

$$
f=\left\{\begin{array}{c}
10 \mathrm{kHz}-\max \omega_{j}(\xi, \mathbf{k}) / 2 \pi \\
\text { if } \min \omega_{j+1}(\xi, \mathbf{k}) / 2 \pi \geq 10 \mathrm{kHz} \\
\left(\min \omega_{j+1}(\xi, \mathbf{k})-\max \omega_{j}(\xi, \mathbf{k})\right) / 2 \pi \\
\text { if } \min \omega_{j+1}(\xi, \mathbf{k}) / 2 \pi \leq 10 \mathrm{kHz}
\end{array}\right.
$$

where, $\max \omega_{j}(\xi, \mathbf{k})$ and $\min \omega_{j}(\xi, \mathbf{k})$ represent the maximum and minimum of the $j^{\text {th }}$ eigenfrequency $\omega_{j}$, respectively, over the $\mathbf{k}$ vector for a given design of the unit cell domain, $\xi$.

We note that this objective function is not strictly differentiable because the location of maximum and minimum eigenvalues over the k-vector may change from one design iteration to another. The problem can be regularized using smooth maximum and minimum functions, as described in the appendix. The results we achieved with the smooth formulation are essentially identical to those achieved with the objective of Eq. (36) and therefore we limit the discussion to a brief comparison in the appendix. It should be noted that in most gradient-based topology optimization studies on band gaps, the issue of differentiability is not discussed and we presume that the results reported in the literature were obtained with standard maximum and minimum operators. One exception we found is the work of Qian \& Sigmund (2011) where a smooth bound formulation is employed. 
The topology optimization problem may be stated as

$$
\begin{array}{ll}
\max _{\xi} & f \\
\text { s.t. : } & \left(K^{*}(\mathbf{k})-\omega^{2} M\right) \tilde{x}_{b}=0, \quad \mathbf{k} \in[\Gamma-\mathrm{X}-\mathrm{M}-\mathrm{K}-\Gamma] \\
& 0 \leq \xi_{e} \leq 1, \quad e=1, \ldots ., N .
\end{array}
$$

\subsection{Design sensitivity analysis}

In order to iteratively update the design variables, we utilize gradient-based optimization, specifically MMA (Svanberg, 1987). This requires the evaluation of the gradient of the objective function with respect to any individual design variable, $\xi^{e}$. The derivative of the objective function $f$ in Eq. (36) with respect to the design variable $\xi^{e}$ is obtained as

$$
\frac{\partial f}{\partial \xi^{e}}= \begin{cases}-\frac{1}{2 \pi} \frac{\partial\left(\max \omega_{n}(\xi, \mathbf{k})\right)}{\partial \xi^{e}}, & \text { if } \min \omega_{n+1}(\xi, \mathbf{k}) / 2 \pi \geq 10 \mathrm{kHz} ; \\ \frac{1}{2 \pi}\left(\frac{\partial\left(\min \omega_{n+1}(\xi, \mathbf{k})\right)}{\partial \xi^{e}}-\frac{\partial\left(\max \omega_{n}(\xi, \mathbf{k})\right)}{\partial \xi^{e}}\right), \quad \text { if } \min \omega_{n+1}(\xi, \mathbf{k}) / 2 \pi \leq 10 \mathrm{kHz} .\end{cases}
$$

For evaluating the objective function derivative in Eq. (38), the derivative of the eigenfrequency with respect to the design variable must be evaluated. For a given wave vector $\mathbf{k}$, the derivative of an eigenfrequency $\omega_{n}$ with respect to the design variable is computed by differentiating Eq. (29) with respect to the design variable $\xi^{e}$, as follows

$$
\frac{\partial\left(\omega_{n}(\xi, \mathbf{k})\right)}{\partial \xi^{e}}=\frac{1}{2 \omega_{n}} \tilde{x}_{b}^{*^{T}}\left(\frac{\partial K^{*}(\mathbf{k})}{\partial \xi^{e}}-\omega_{n}^{2} \frac{\partial M}{\partial \xi^{e}}\right) \tilde{x}_{b}^{*},
$$

where, $\tilde{x}_{b}^{*}$ is the global mass normalized eigenvector, and the derivative of the condensed stiffness matrix $K^{*}$ with respect to the design variable is obtained as

$$
\begin{aligned}
\frac{\partial K^{*}}{\partial \xi^{e}}= & \frac{\partial K^{m m}}{\partial \xi^{e}}-\frac{\partial K^{m e}}{\partial \xi^{e}}\left(K^{e e}\right)^{-1} K^{e m}+ \\
& K^{m e}\left(K^{e e}\right)^{-2} K^{e m} \frac{\partial K^{e e}}{\partial \xi^{e}}-K^{m e}\left(K^{e e}\right)^{-1} \frac{\partial K^{e m}}{\partial \xi^{e}}
\end{aligned}
$$

The derivatives of the stiffness and the mass matrices $\frac{\partial K^{m m}}{\partial \xi^{e}}, \frac{\partial K^{m e}}{\partial \xi^{e}}, \frac{\partial K^{e m}}{\partial \xi^{e}}, \frac{\partial K^{e e}}{\partial \xi^{e}}$, and $\frac{\partial M}{\partial \xi^{e}}$ in Eqs. (39)-(40), are evaluated using Eqs. (21)-(25) as follows

$$
\begin{gathered}
\frac{\partial K^{m m}}{\partial \xi^{e}}=\int_{\mathcal{B}_{t}^{e}} \frac{\partial \tilde{\mu}^{e}}{\partial \xi^{e}} \frac{\partial N_{a}}{\partial x_{j}} \frac{\partial N_{b}}{\partial x_{j}} d s, \\
\frac{\partial K^{m e}}{\partial \xi^{e}}=-\int_{\mathcal{B}_{t}^{e}} \frac{\partial \tilde{d}^{e}}{\partial \xi^{e}} \frac{\partial N_{a}}{\partial x_{j}} \frac{\partial N_{b}}{\partial x_{j}} d s,
\end{gathered}
$$




$$
\begin{gathered}
\frac{\partial K^{e m}}{\partial \xi^{e}}=-\int_{\mathcal{B}_{t}^{e}} \frac{\partial \tilde{d}^{e}}{\partial \xi^{e}} \frac{\partial N_{a}}{\partial x_{j}} \frac{\partial N_{b}}{\partial x_{j}} d s \\
\frac{\partial K^{e e}}{\partial \xi^{e}}=-\int_{\mathcal{B}_{t}^{e}} \frac{\partial \varepsilon^{e}}{\partial \xi^{e}} \frac{\partial N_{a}}{\partial x_{j}} \frac{\partial N_{b}}{\partial x_{j}} d s, \\
\frac{\partial M}{\partial \xi^{e}}=\int_{\mathcal{B}_{t}^{e}} \frac{\partial \rho^{e}}{\partial \xi^{e}} N_{a} N_{b} d s,
\end{gathered}
$$

${ }_{263}$ where $\frac{\partial J_{m}^{e}}{\partial \xi^{e}}=J_{m}^{(f)}-J_{m}^{(m)}$, and the derivative of the stretch parameter with respect of design 264 variable $\frac{\partial \lambda}{\partial \xi^{e}}$ is evaluated by differentiating Eq. (13) with respect to $\xi^{e}$ and expressed as $\frac{\partial \lambda}{\partial \xi_{e}}=\frac{\left[\left(\varepsilon^{(f)}-\varepsilon^{(m)}\right) e_{3}^{2}-\left(\lambda^{2}-\lambda^{-4}\right)\left(\frac{\mu^{e}\left(J_{m}^{(f)}-J_{m}^{(m)}\right)+J_{m}^{e}\left(\mu^{(f)}-\mu^{(m)}\right)}{\left(J_{m}^{e}-2 \lambda^{2}-\lambda^{-4}+3\right)}+\frac{\mu^{e} J_{m}^{e}\left(J_{m}^{(f)}-J_{m}^{(m)}\right)}{\left(J_{m}^{e}-2 \lambda^{2}-\lambda^{-4}+3\right)^{2}}\right)\right]}{\left[\left(2 \lambda^{2}+4 \lambda^{-5}\right)\left(\sum_{e=1}^{N} \mu^{e}\right)+\left(\lambda^{2}-\lambda^{-4}\right)\left(4 \lambda-4 \lambda^{-5}\right)\left(\sum_{e=1}^{N} \frac{\mu^{e} J_{m}^{e}}{\left(J_{m}^{e}-2 \lambda^{2}-\lambda^{-4}+3\right)^{2}}\right)\right]}$

\footnotetext{
${ }^{1}$ Note that the expression (46) corresponds to $\tilde{\mu}^{e}$ and $\xi^{e}$ of the same finite element. However, in the evaluation of $\tilde{\mu}^{e}$ there exists a weak coupling to other elements due to the solution of $\lambda$ that is a global state variable. Hence, for two distinct elements denoted $e_{1}$ and $e_{2}$, the derivative is

$$
\frac{\partial \tilde{\mu}^{e_{1}}}{\partial \xi^{e_{2}}}=\frac{2 \mu^{e_{1}} J_{m}^{e_{1}} \lambda \frac{\partial \lambda}{\partial \xi^{e_{2}}}}{\left(J_{m}^{e_{1}}-2 \lambda^{2}-\lambda^{-4}+3\right)}-\frac{\mu^{e_{1}} J_{m}^{e_{1}} \lambda^{2}\left[-4 \lambda \frac{\partial \lambda}{\partial \xi^{e_{2}}}+4 \lambda^{-5} \frac{\partial \lambda}{\partial \xi^{e_{2}}}\right]}{\left(J_{m}^{e_{1}}-2 \lambda^{2}-\lambda^{-4}+3\right)^{2}}
$$

These coupling terms are several orders of magnitude smaller than the terms of (46) and were found to have no influence on the optimized design. Hence they are omitted from subsequent derivations.
} 


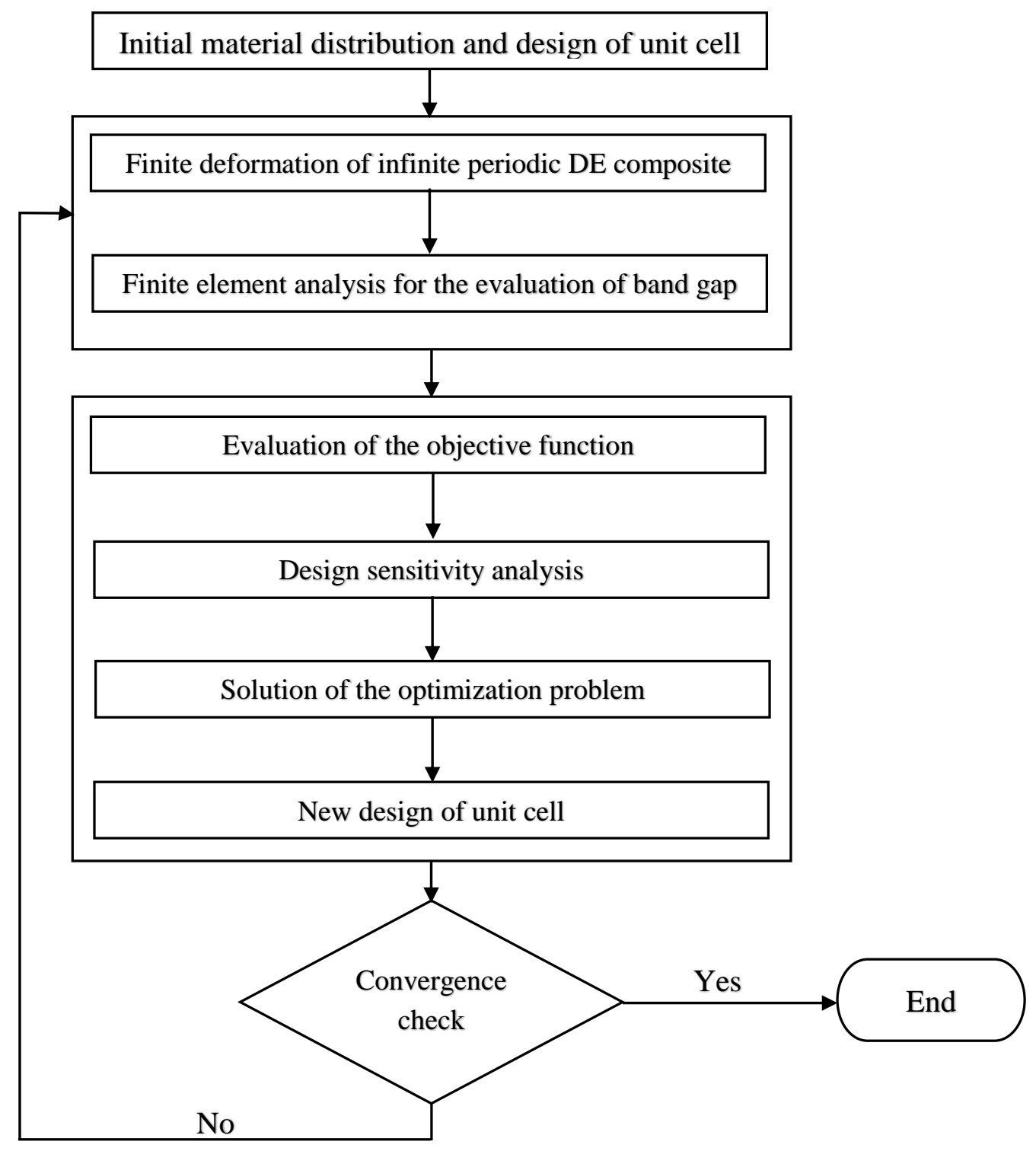

Figure 4: A schematic representation of the optimization process.

\subsection{Implementation}

The topology optimization framework presented in this paper is implemented by developing an in-house MATLAB code. The topology optimization process starts with generating one quarter of a twice-symmetric structure of the unit cell. In order to generate a symmetric unit cell, we randomly distribute design variables in one quarter of the unit cell, and assign these design variables symmetrically in the other three quarters. We note that this initial unit cell structure may not have gaps in its spectrum. Then, based on the finite element framework introduced in Section 3, we obtain the band diagram and the corresponding eigenvectors. Accordingly, the objective function is evaluated. Subsequently, we evaluate the gradient of the objective with respect to the design variables using Eq. (38). According to the evaluated objective function gradients, MMA proposes a new vector of design variables by solving a convex subproblem. This yields the formation of a 
Table 1: Material properties.

\begin{tabular}{ccc}
\hline \hline & \multicolumn{2}{c}{ Material } \\
\cline { 2 - 3 } & Silicone CF19-2186 & Polyurethane PT6100S \\
\hline Shear modulus $\mu(\mathrm{kPa})$ & 333 & 5667 \\
Locking parameter $J_{m}$ & 46.3 & 6.67 \\
Relative permittivity $\epsilon_{r}$ & 2.8 & 7 \\
Density $\rho\left(\mathrm{kg} / \mathrm{m}^{3}\right)$ & 1100 & 1200 \\
\hline \hline
\end{tabular}

new structure with the updated design variables. Thereafter, we repeat the finite element analysis for the evaluation of band gaps and MMA optimization scheme for updating the design variables. Such an iterative process continues until the maximum change in any design variable is less than $10^{-6}$. Figure 4 depicts schematic representation of the iterative optimization process.

\section{Numerical results and discussions}

In this section, we provide the numerical results for the phononic band gaps in dielectric elastomers designed by using the topology optimization framework presented in the previous section. The dielectric elastomer composite is assumed to be composed of polyurethane PT6100S fibers embedded in the silicone CF19-2186 matrix (Getz et al., 2017). The properties of these materials are listed in Table 1. In the undeformed configuration, we set the lattice parameter or the size of the square unit cell to be $A=6.3 \mathrm{~mm}$. In order to discretize the unit cell, we used the four nodes, linear quadrilateral finite elements with two degrees of freedom (one anti-plane displacement and one electric potential) per node.

\subsection{Validation of the finite element framework}

First, for validating the finite element framework presented in Section 4 and investigating the influence of the bias electric field on the band structure, we compute the performance of a standard unit cell composed of circular fibers embedded in the matrix, as shown in Fig. $5 \mathrm{k}$. The volume fraction of fibers in the unit cell is taken to be 0.5 . The unit cell is discretized by 1139 quadrilateral finite elements as shown in Fig. $5 \mathrm{k}$ (left). In all the plots in this paper, the yellow color denotes the fiber and the blue color denotes the matrix. For investigating the effect of the bias electric field on the band diagram, we perform two cases, one without bias electric field $\left(e_{3}=0\right)$ and the other with bias electric field $e_{3}=300 \mathrm{MV} / \mathrm{m}$. For both cases, without and with bias electric field, Figs. $5 \mathrm{~b}$ and 5c, respectively, compare the band structure computed using the finite element framework presented in this work and the plane wave expansion method (PWE) (Shmuel, 2013). The plot shows a very good agreement between FEM and PWE, validating our finite element framework. From the band diagrams, it is evident that the width of the widest gap at $e_{3}=0$, i.e., $(\Delta \omega / 2 \pi=0.7626 \mathrm{kHz})$, is smaller than that at $e_{3}=300 \mathrm{MV} / \mathrm{m}$, i.e., $(\Delta \omega / 2 \pi=0.8073 \mathrm{kHz})$, denoting an increase in the band gap width of $5.86 \%$. This example suggests that the bias electric field has a favorable impact as it improves the maximization of band gap widths.

\subsection{Topology optimization results}

In this section, first, we present the topologies for the maximization of the band gap width between the first four adjacent bands (i.e., $1^{\text {st }}$ and $2^{\text {nd }}$ bands, $2^{\text {nd }}$ and $3^{\text {rd }}$ bands, $3^{\text {rd }}$ and $4^{\text {th }}$ bands, 
(a)
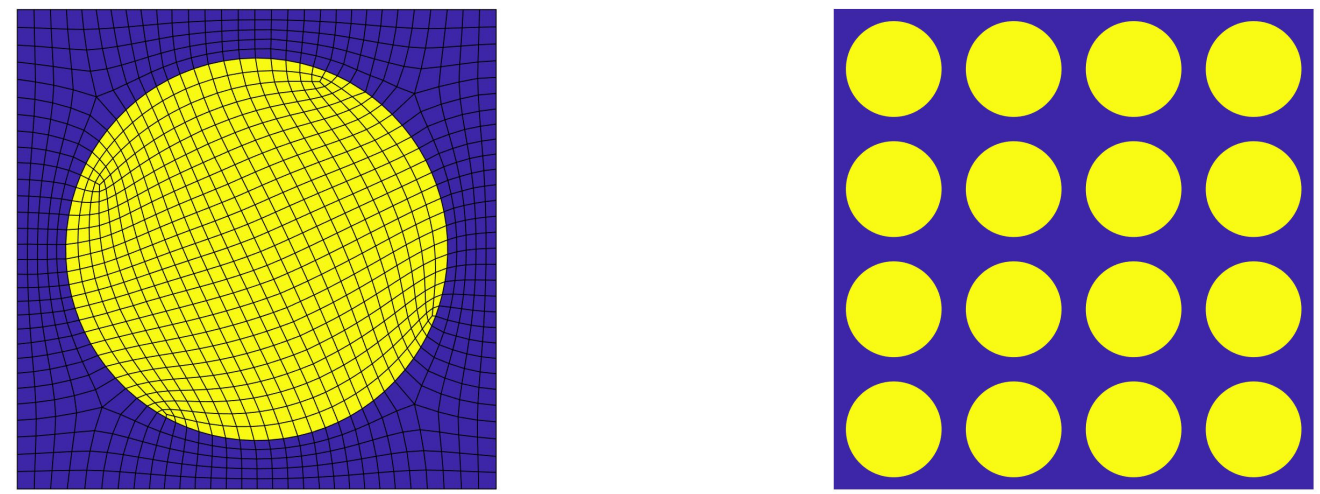

(b)

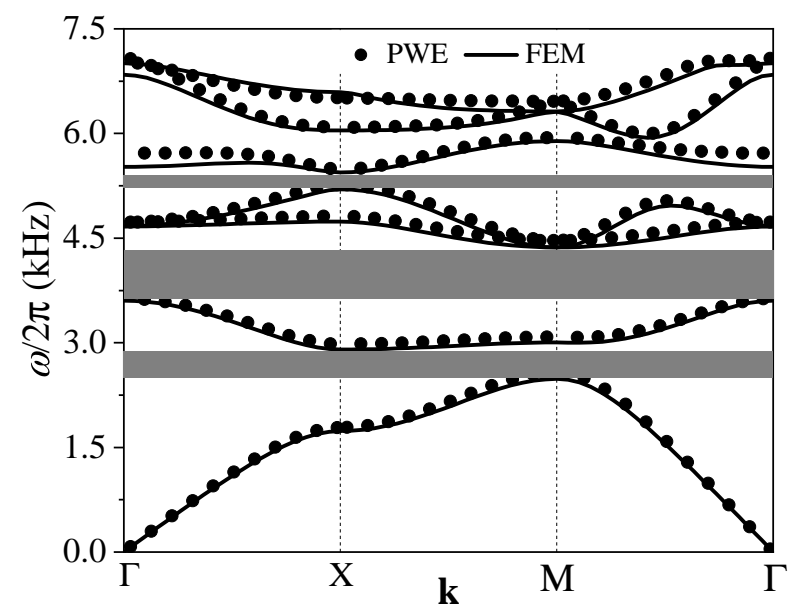

(c)

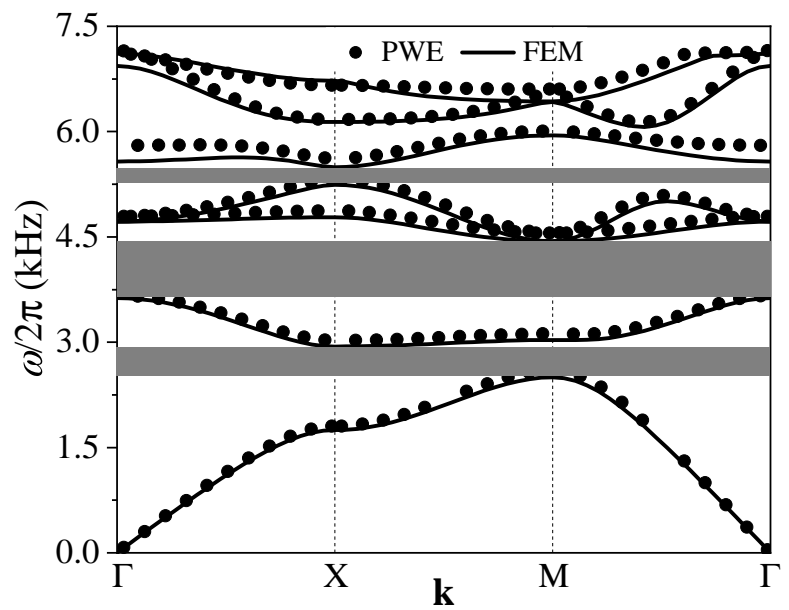

Figure 5: Influence of the bias electric field on the band structure of a DE composite with circular fiber and comparison of FEM results (solid lines) with the plane wave expansion method (PWE) results (symbols): (a) Unit cell (left) and periodic matrix (right), band diagrams (b) when bias electric field $e_{3}=0$, and (c) when bias electric field $e_{3}=300 \mathrm{MV} / \mathrm{m}$.

$\& 4^{\text {th }}$ and $5^{\text {th }}$ bands) and identify the two adjacent bands that have the largest optimized band gap in the audible frequency range. For these four optimization cases, we consider two values of the applied electric field, i.e., $e_{3}=150 \mathrm{MV} / \mathrm{m}$ and $e_{3}=300 \mathrm{MV} / \mathrm{m}$, and discretize the unit cell by $20 \times 20$, bi-linear quadrilateral elements. The initial material distribution of the unit cells is taken to be random. The resulting designs of the unit cells that optimize the band gap widths between the first four adjacent bands at applied electric field $e_{3}=150 \mathrm{MV} / \mathrm{m}$ and $e_{3}=300 \mathrm{MV} / \mathrm{m}$ are depicted in the left panels of Figs. 6 and 7, respectively. In both figures, the middle panels represent the periodic matrices composed of four optimized unit cells, while the right panels show the band diagrams corresponding to the optimized unit cells depicted in the left panels. The estimates of the optimized band gaps between the first four adjacent bands and the corresponding fiber volume fractions for both the applied electric field values are listed in Table 2. From Table 2 and the left panels of Figs. 6 and 7, we observe the maximum band gap width when the band gap is optimized between the $4^{\text {th }}$ and $5^{\text {th }}$ bands. At $e_{3}=150 \mathrm{MV} / \mathrm{m}$, the value of the maximal band gap width in 
Table 2: Optimized band gaps and fiber volume fractions, when maximizing gaps between two different adjacent bands.

\begin{tabular}{|c|c|c|c|c|}
\hline \multirow[t]{2}{*}{ Bias electric field $\left(e_{3}\right) \rightarrow$} & \multicolumn{2}{|c|}{$150 \mathrm{MV} / \mathrm{m}$} & \multicolumn{2}{|c|}{$300 \mathrm{MV} / \mathrm{m}$} \\
\hline & Band gap & Fiber fraction & Band gap & Fiber fraction \\
\hline $1^{\text {st }}$ and $2^{\text {nd }}$ bands & 1.58 & 0.65 & 1.71 & 0.66 \\
\hline $2^{\text {nd }}$ and $3^{\text {rd }}$ bands & 1.94 & 0.58 & 2.25 & 0.57 \\
\hline $3^{\text {rd }}$ and $4^{\text {th }}$ bands & 1.73 & 0.57 & 2.08 & 0.58 \\
\hline $4^{\text {th }}$ and $5^{\text {th }}$ bands & 4.31 & 0.67 & 4.61 & 0.68 \\
\hline
\end{tabular}

the audible frequency range is $4.31 \mathrm{kHZ}$ and the corresponding fiber volume fraction is 0.67 . The maximal band gap width achieved in the audible frequency range at $e_{3}=300 \mathrm{MV} / \mathrm{m}$, is $4.61 \mathrm{kHZ}$ with 0.68 fiber volume fraction.

Next, for the identified adjacent bands (i.e., $4^{\text {th }}$ and $5^{\text {th }}$ bands) representing maximum optimized band gap in the audible frequency range, we present the optimized designs for different initial layouts of the unit cells. We consider three initial layouts of the unit cells (fully random, single central square fiber inclusion, and fiber inclusions at multiple locations) as shown in Table 3 . For these three initial layouts, the iteration history of the objective function and the evolution of the optimal topology of the unit cells at applied electric fields $e_{3}=150 \mathrm{MV} / \mathrm{m}$ and $e_{3}=300 \mathrm{MV} / \mathrm{m}$ are represented in Figs. 8 and 9 , respectively. In both figures, the left columns show the iteration history of the objective function and the evolution of the unit cell structure for a random initial material distribution; the middle columns show the same data for an initial design consisting of a central square fiber inclusion; and the right columns show the same data for an initial design with fiber inclusions at multiple locations. From Figs. 8 and 9, we observe that the optimized topologies depend on the initial layouts. This phenomenon of dependency on the initial layouts for the unconstrained band gap maximization problem has been previously described in the literature (Yi et al., 2019; Li et al., 2016b) and is somewhat expected in gradient-based optimization. From the plots of the iteration histories of the objective function, we observe that the objective function increases rapidly at the starting stage of the topology optimization process and then the convergence rate becomes significantly slowed down over the iterations. The estimates of the optimized band gaps and the corresponding fiber volume fractions for all three initial layouts and both the values of the applied electric field are listed in Table 3. As evident from Table 3, the third initial guess (fiber inclusions at multiple locations) for the unit cell layout is best among all the three initial guesses, as it shows the maximum optimized band gap for both values of the applied electric fields. The maximum band gap achieved at $e_{3}=150 \mathrm{MV} / \mathrm{m}$ is $4.37 \mathrm{kHz}$ and that at $e_{3}=300 \mathrm{MV} / \mathrm{m}$ is equal to $4.61 \mathrm{kHz}$. The fiber volume fractions corresponding to optimized layouts are the same for both applied electric field and are equal to 0.68 . As evident from the convergence history plots, for the third initial layout of the unit cell, the topology optimization process converges faster in comparison to the other two initial layouts. This is expected because it utilizes an educated initial guess.

Next, we investigate the dependence of the optimized band gaps and the unit cell designs on the FE mesh resolution, that affects the design resolution. For this purpose, we study the maximization of band gap width between the $4^{\text {th }}$ and $5^{\text {th }}$ bands (i.e., having the highest band gaps in the audible frequency range). For mesh dependency analyses, we discretize the unit cell by $20 \times 20$, 
(a)
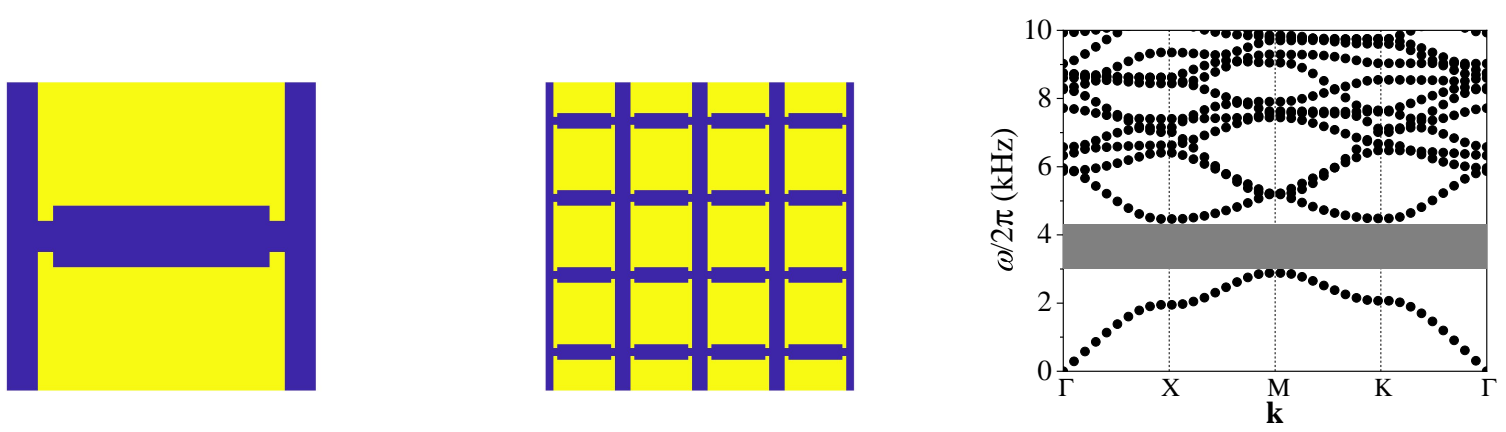

(b)
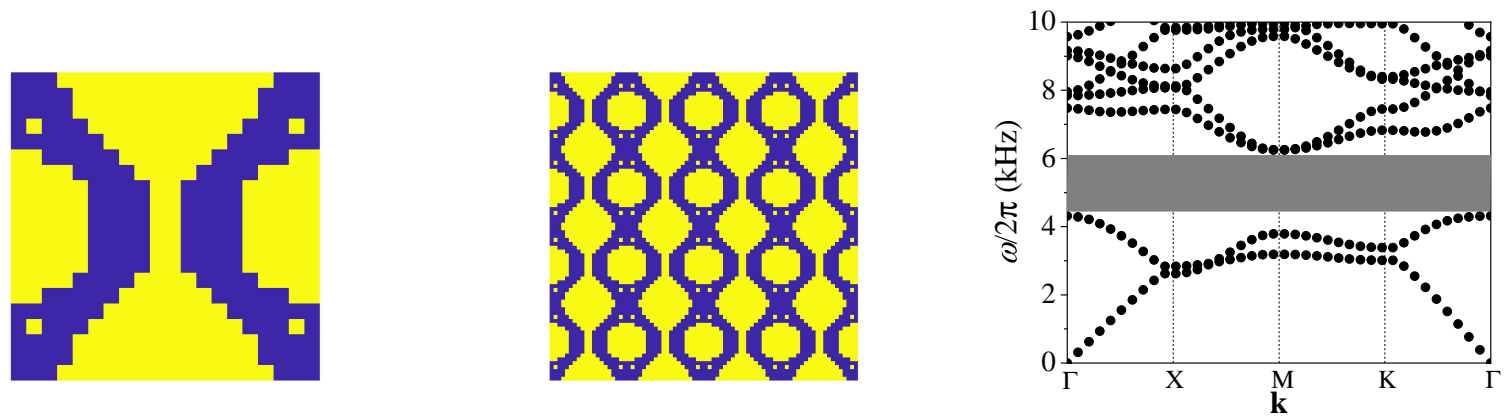

(c)
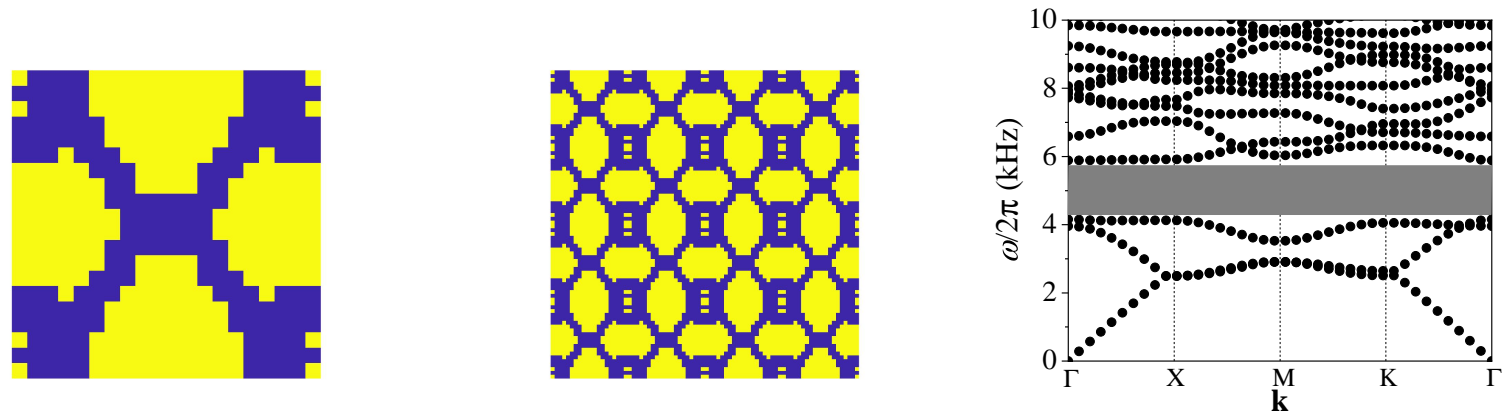

(d)
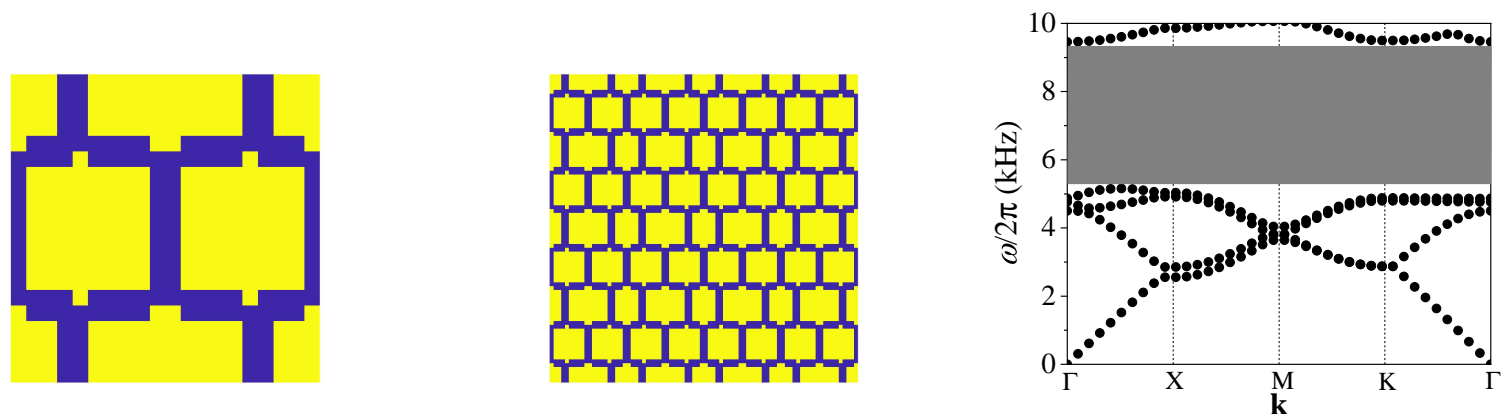

Figure 6: Unit cells (left), periodic matrices composed of $4 \times 4$ unit cells (middle), and band diagrams (right) of the optimized results when maximizing the band gap between (a) $1^{\text {st }}$ and $2^{\text {nd }}$ bands, (b) $2^{\text {nd }}$ and $3^{\text {rd }}$ bands, (c) $3^{\text {rd }}$ and $4^{\text {th }}$ bands, and (d) $4^{\text {th }}$ and $5^{\text {th }}$ bands, when bias electric field $e_{3}=150 \mathrm{MV} / \mathrm{m}$.

maximizing the band gaps at the aforementioned two values of the applied electric field. For all 
(a)
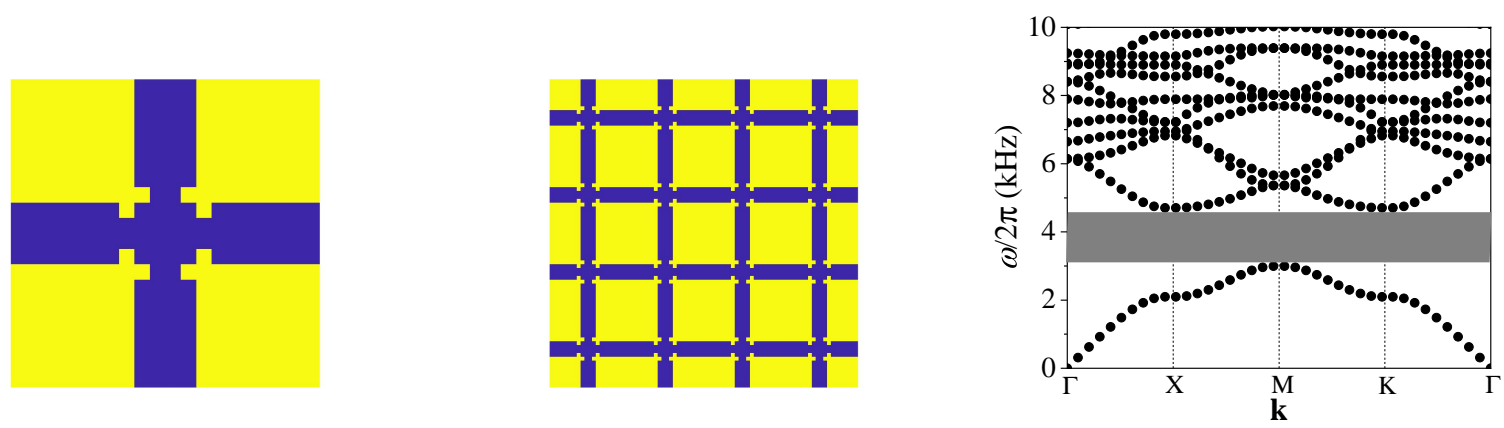

(b)
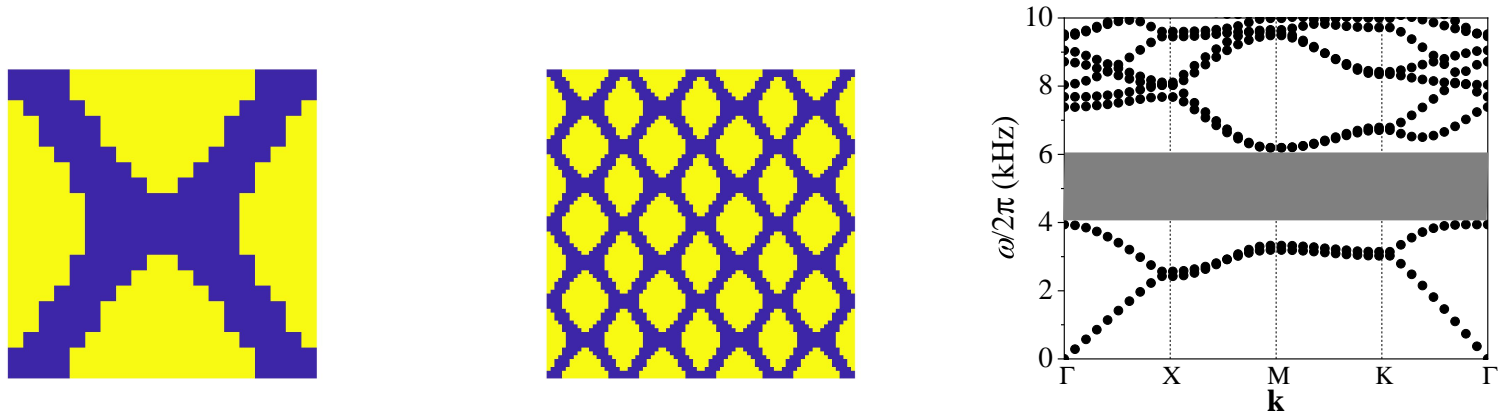

(c)
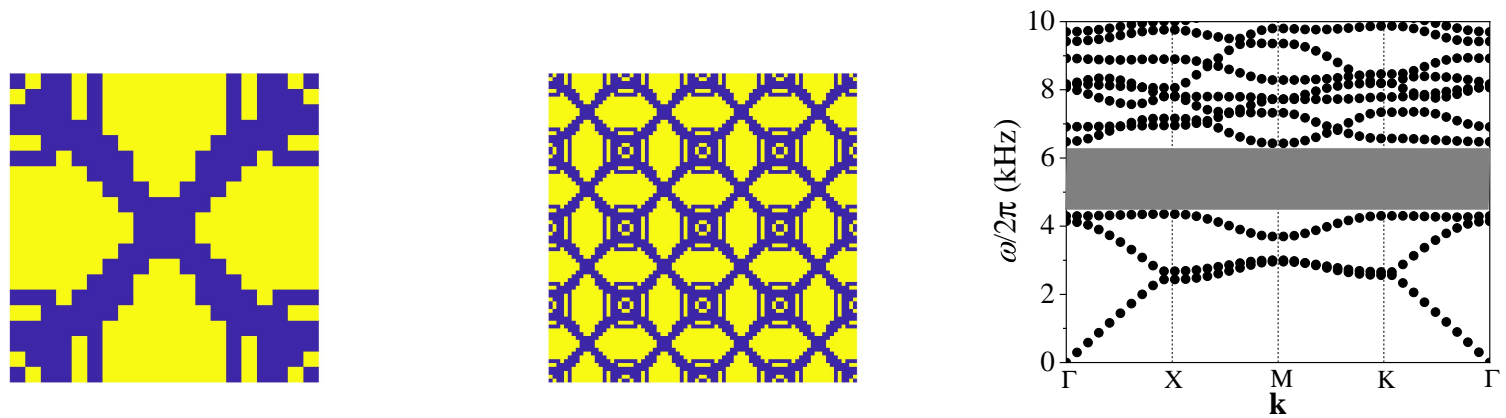

(d)
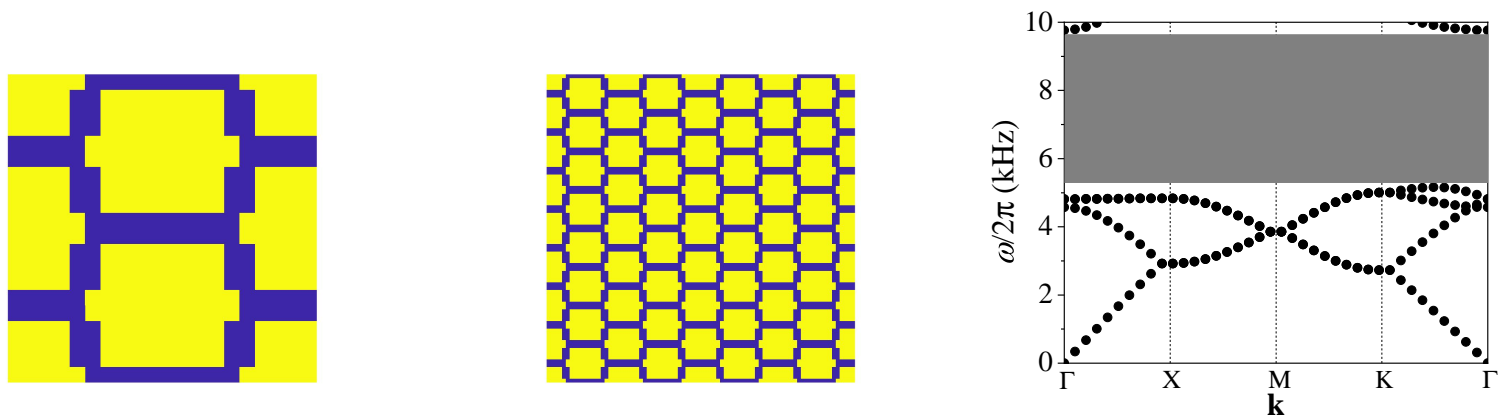

Figure 7: Unit cells (left), periodic matrices composed of $4 \times 4$ unit cells (middle), and band diagrams (right) of the optimized results when maximizing the band gap between (a) $1^{\text {st }}$ and $2^{\text {nd }}$ bands, (b) $2^{\text {nd }}$ and $3^{\text {rd }}$ bands, (c) $3^{\text {rd }}$ and $4^{\text {th }}$ bands, and (d) $4^{\text {th }}$ and $5^{\text {th }}$ bands, when bias electric field $e_{3}=300 \mathrm{MV} / \mathrm{m}$.

three mesh densities, we consider the same initial layout of the unit cell, i.e., the third layout of the aforementioned discussion for which we obtained the maximum at both the applied electric fields. 
Table 3: Effect of initial material layout on the optimized band gaps and fiber volume fractions for maximization of band gap between $4^{\text {th }}$ and $5^{\text {th }}$ bands.

\begin{tabular}{|c|c|c|c|c|c|c|}
\hline \multirow{2}{*}{$\begin{array}{l}\text { Initial material layout } \rightarrow \\
\text { Bias electric field }\left(e_{3}\right)\end{array}$} & \multicolumn{2}{|c|}{$\begin{array}{l}x=4 \\
x-4\end{array}$} & \multirow[b]{2}{*}{ Band gap } & \multirow[b]{2}{*}{ Fiber fraction } & \multirow[b]{2}{*}{ Band gap } & \multirow[b]{2}{*}{ Fiber fraction } \\
\hline & Band gap & Fiber fraction & & & & \\
\hline $\begin{array}{l}150 \mathrm{MV} / \mathrm{m} \\
300 \mathrm{MV} / \mathrm{m}\end{array}$ & $\begin{array}{l}4.31 \mathrm{kHz} \\
4.61 \mathrm{kHz}\end{array}$ & $\begin{array}{l}0.67 \\
0.68\end{array}$ & $\begin{array}{l}4.30 \mathrm{kHz} \\
4.51 \mathrm{kHz}\end{array}$ & $\begin{array}{l}0.68 \\
0.68\end{array}$ & $\begin{array}{l}4.37 \mathrm{kHz} \\
4.61 \mathrm{kHz}\end{array}$ & $\begin{array}{l}0.68 \\
0.68\end{array}$ \\
\hline
\end{tabular}

Table 4: Effect of mesh size on the optimized band gaps and fiber volume fractions for maximization of band gap between $4^{\text {th }}$ and $5^{\text {th }}$ bands.

\begin{tabular}{|c|c|c|c|c|c|c|}
\hline \multirow{2}{*}{$\begin{array}{c}\text { Mesh density } \rightarrow \\
\text { Bias electric field }\left(e_{3}\right)\end{array}$} & \multicolumn{2}{|c|}{$20 \times 20$} & \multicolumn{2}{|c|}{$30 \times 30$} & \multicolumn{2}{|c|}{$40 \times 40$} \\
\hline & Band gap & Fiber fraction & Band gap & Fiber fraction & Band gap & Fiber fraction \\
\hline $\begin{array}{l}150 \mathrm{MV} / \mathrm{m} \\
300 \mathrm{MV} / \mathrm{m}\end{array}$ & $\begin{array}{l}4.37 \mathrm{kHz} \\
4.61 \mathrm{kHz}\end{array}$ & $\begin{array}{l}0.680 \\
0.680\end{array}$ & $\begin{array}{l}4.33 \mathrm{kHz} \\
4.56 \mathrm{kHz}\end{array}$ & $\begin{array}{l}0.715 \\
0.706\end{array}$ & $\begin{array}{l}4.30 \mathrm{kHz} \\
4.53 \mathrm{kHz}\end{array}$ & $\begin{array}{l}0.680 \\
0.680\end{array}$ \\
\hline
\end{tabular}

The optimized designs of the unit cell and the corresponding band diagrams for $20 \times 20,30 \times 30$, and $40 \times 40 \mathrm{mesh}$ densities and at $e_{3}=150 \mathrm{MV} / \mathrm{m}$ are compared in the left, middle and right panels of Fig. 10a, respectively. For both values of bias electric field, the estimates of the optimized band gaps and the corresponding fiber volume fractions at the aforementioned three mesh densities are listed in Table 4 . From the table, it is evident that the value of the optimized band gap decreases with an increase in the mesh density for both the values of the bias electric field. This is attributed to the effect of FE mesh refinement on the evaluation of the band gap. Interestingly, at $20 \times 20$ and $40 \times 40$ mesh density, we obtain the same designs of the unit cell and fiber volume fractions for both values of the applied electric field.

Further, for a one-to-one comparison of the optimized unit cell structures obtained at different mesh sizes, we evaluate the band gaps for all three optimized structures on a $120 \times 120$ mesh resolution. This resolution is chosen such that all optimized designs can be projected precisely to a common mesh. Table 5 enlists the band gap values evaluated on a $120 \times 120$ mesh resolution for the optimized unit cells obtained at the aforementioned three mesh densities and two values of bias electric field. The band diagrams evaluated at $120 \times 120$ mesh resolution of the unit cells optimized at three mesh sizes are shown in Fig. 11. From Table 5, we can see that as the design resolution increases, so does the width of the optimized band gap. This demonstrates the efficacy of the topology optimization method: better performance can be achieved by increasing the design resolution, hence expanding the design space. The unit cell layouts obtained from the optimization at $40 \times 40$ mesh density show the maximum band gap for both bias electric fields. For $120 \times 120$ mesh resolution, the value of maximum band gap achieved at $e_{3}=150 \mathrm{MV} / \mathrm{m}$ is $4.24 \mathrm{kHz}$ with 0.68 fiber volume fraction and that at $e_{3}=300 \mathrm{MV} / \mathrm{m}$ is equal to $4.44 \mathrm{kHz}$ with the same fiber volume fraction of 0.68 .

Finally, we discuss the advantages of the gradient-based topology optimization framework developed in this paper over the method based on PWE and a genetic algorithm (GA) for maximization 
(a)
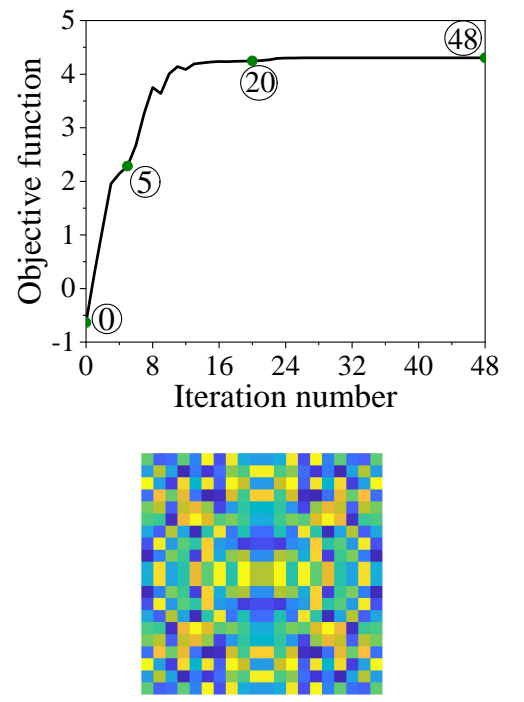

Initial state (Iteration=0)

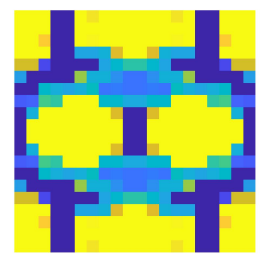

Intermediate state (Iteration=5)

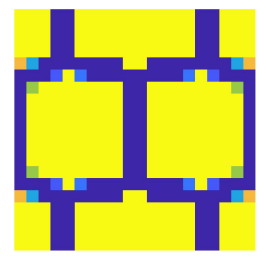

Intermediate state $($ Iteration $=20)$

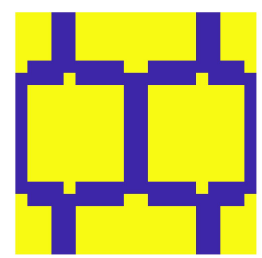

Final state (Iteration=48) (b)
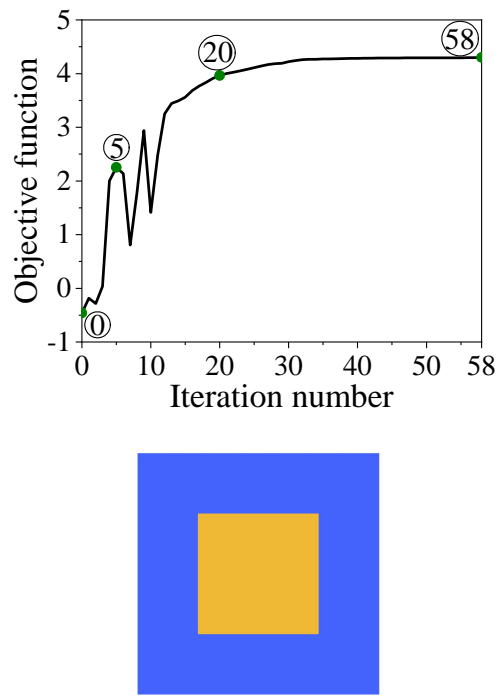

Initial state (Iteration $=0)$

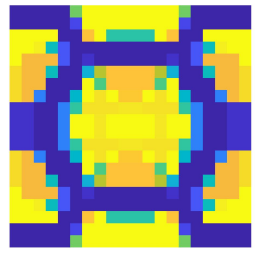

Intermediate state (Iteration=5)

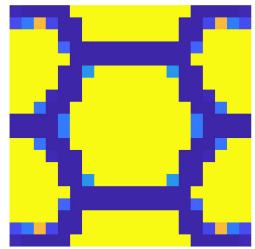

Intermediate state (Iteration=20)

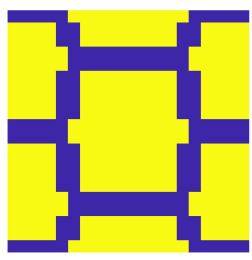

Final state $($ Iteration $=58)$ (c)
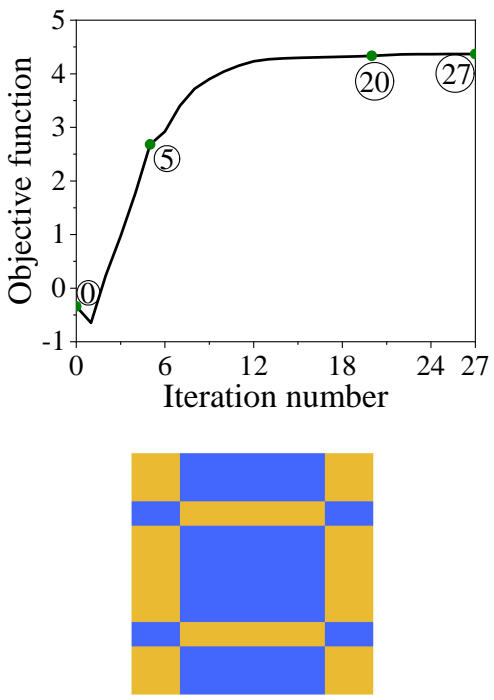

Initial state (Iteration $=0$ )

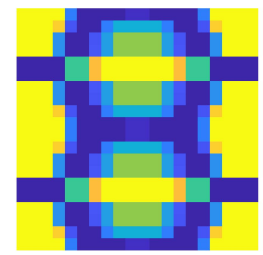

Intermediate state (Iteration=5)

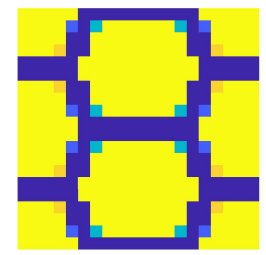

Intermediate state $($ Iteration $=20)$

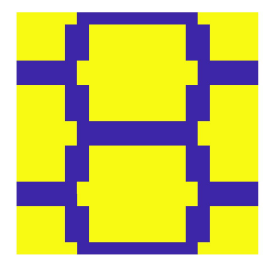

Final state $($ Iteration=27)

Figure 8: Optimization history and the layout evolution history of the unit cells for maximizing the band gap between $4^{\text {th }}$ and $5^{\text {th }}$ bands at bias electric field $e_{3}=150 \mathrm{MV} / \mathrm{m}$, for various initial layouts of the unit cells: (a) fully random; (b) central square fiber inclusion; and (c) fiber inclusions at multiple locations.

of band gaps in dielectric elastomers (Bortot et al., 2018). For this purpose, we compare the optimized designs of the unit cells and the corresponding optimized band gaps obtained using 
(a)
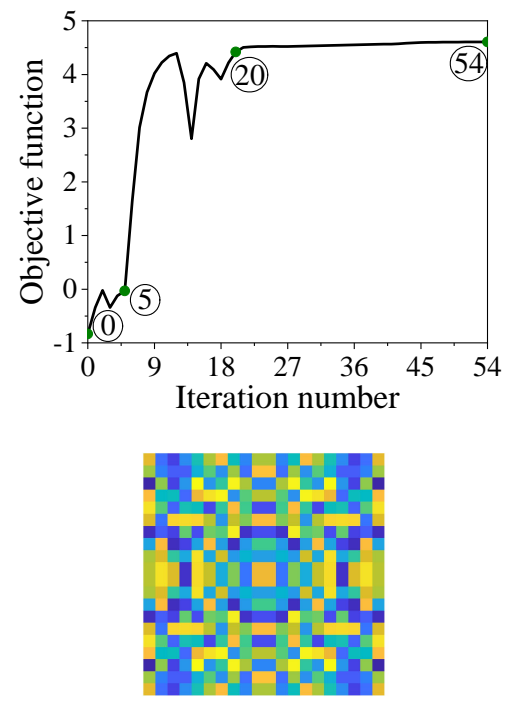

Initial state (Iteration=0)

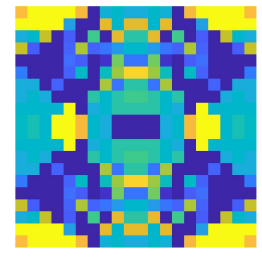

Intermediate state (Iteration=5)

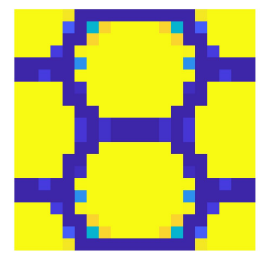

Intermediate state $($ Iteration $=20)$

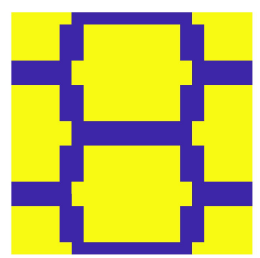

Final state (Iteration=54) (b)
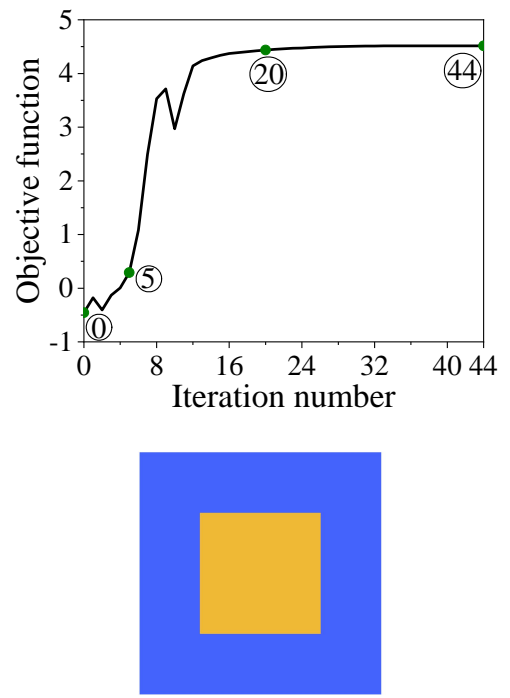

Initial state (Iteration=0)

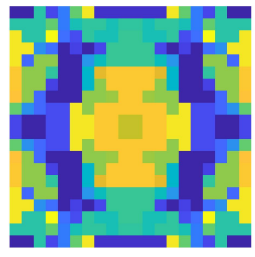

Intermediate state (Iteration=5)

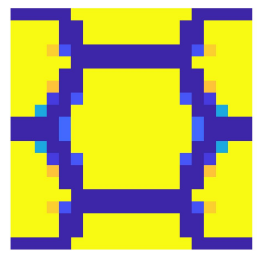

Intermediate state (Iteration=20)

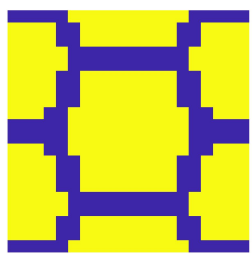

Final state $($ Iteration $=44)$ (c)
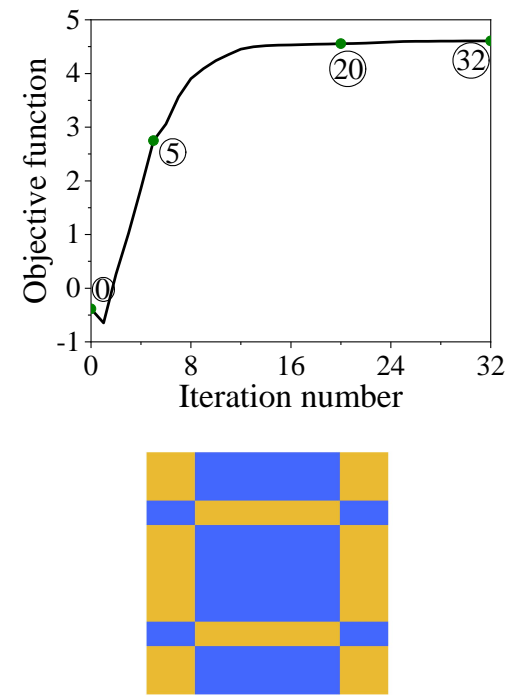

Initial state (Iteration $=0$ )

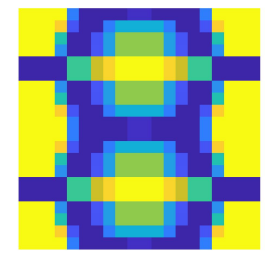

Intermediate state $($ Iteration $=5)$

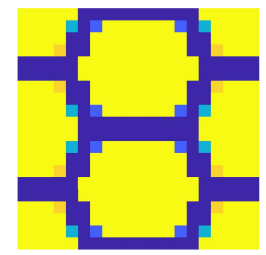

Intermediate state $($ Iteration $=20)$

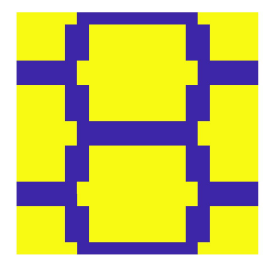

Final state $($ Iteration=32)

Figure 9: Optimization history and the layout evolution history of the unit cells for maximizing the band gap between $4^{\text {th }}$ and $5^{\text {th }}$ bands at bias electric field $e_{3}=300 \mathrm{MV} / \mathrm{m}$, for various initial layouts of the unit cells: (a) fully random; (b) central square fiber inclusion; and (c) fiber inclusions at multiple locations.

both methods of optimization. For one-to-one comparison, we consider the design structures optimized at the same mesh density i.e., $20 \times 20$. Figures $12 \mathrm{a}$ and $12 \mathrm{~b}$ show the designs of the 
(a)
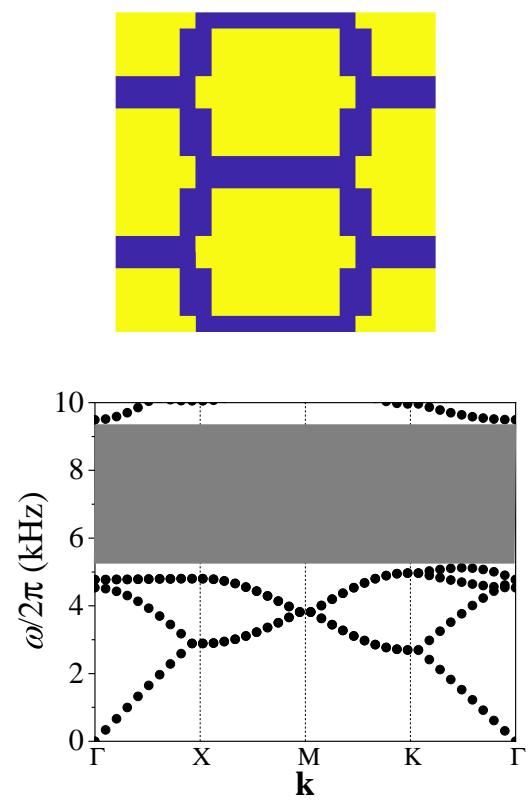

(b)
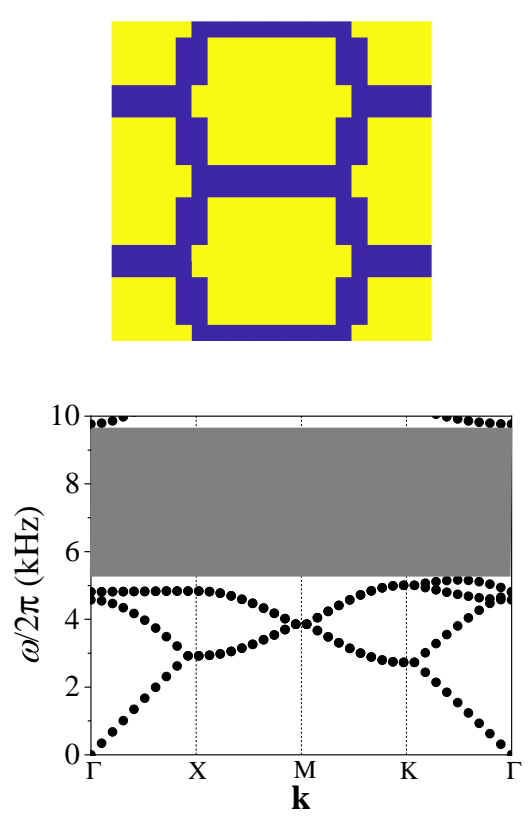
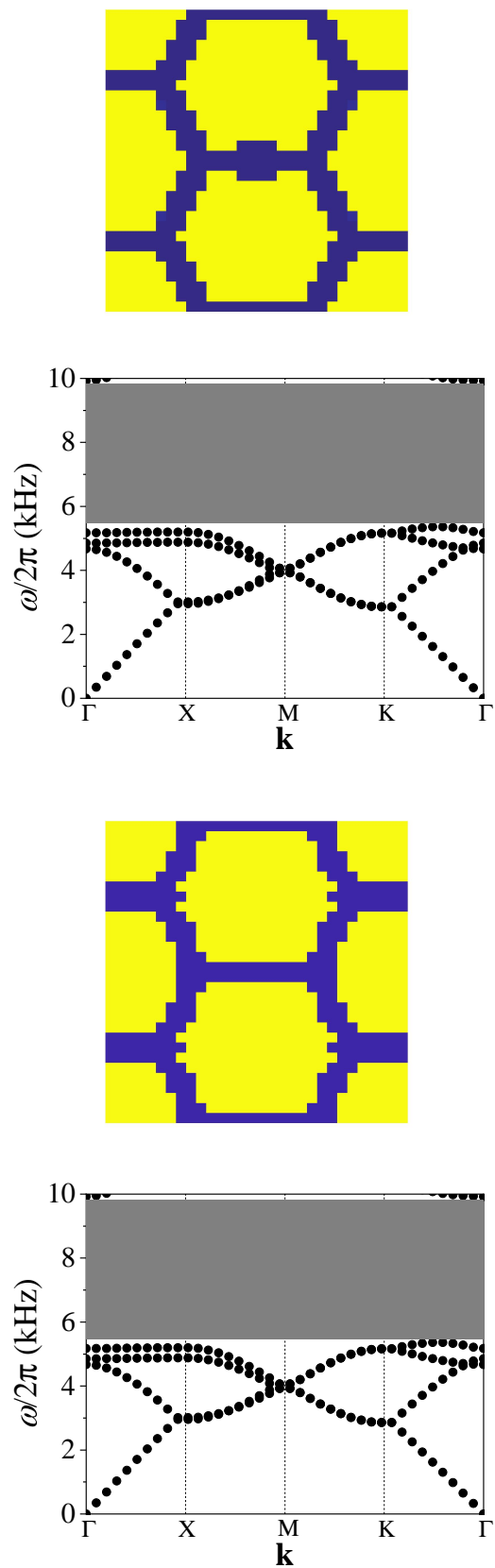

$40 \times 40$
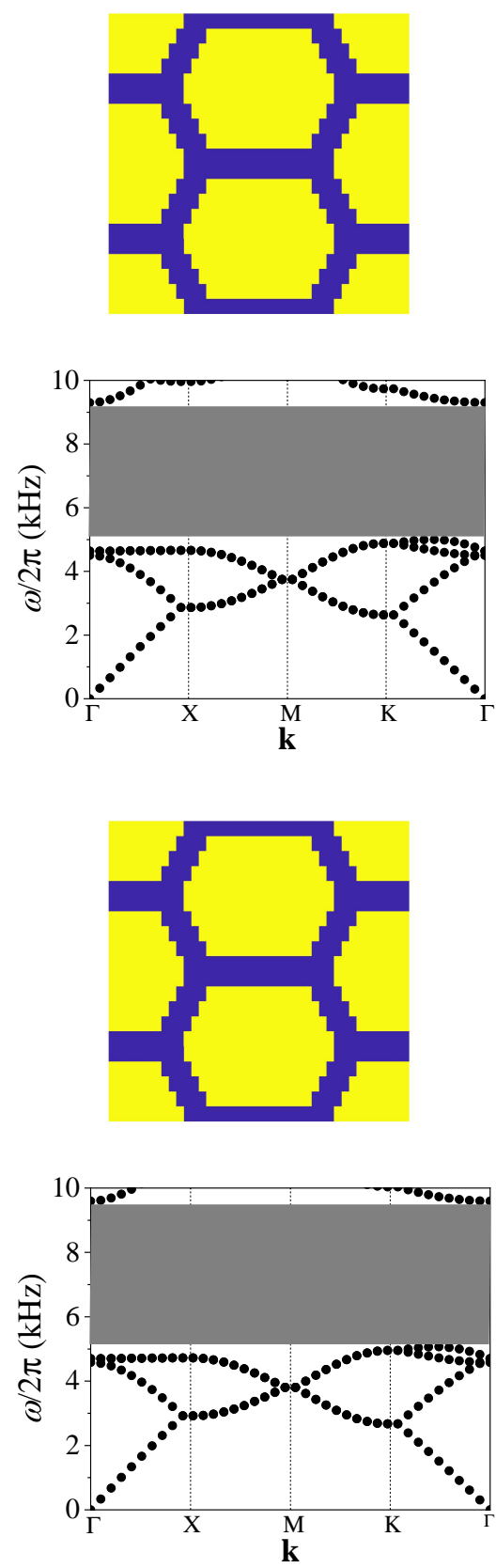

Figure 10: Effect of mesh size (left, $20 \times 20$; middle, $30 \times 30$; right, $40 \times 40$ ) on the optimized layouts of the unit cells for maximizing the band gap between $4^{\text {th }}$ and $5^{\text {th }}$ bands, when bias electric field (a) $e_{3}=150 \mathrm{MV} / \mathrm{m}$, and (b) $e_{3}=300 \mathrm{MV} / \mathrm{m}$.

optimized unit cell obtained using gradient-based and GA-based topology optimization methods, respectively, at bias electric field $e_{3}=150$. The fiber volume fraction for the unit cell optimized using a gradient-based optimization scheme is 0.68 and while for that optimized using GA is equal to 0.64 . The band diagrams for optimized unit cells in Figs. $12 \mathrm{a}$ and $12 \mathrm{p}$ are shown in Fig. 12k. From the band diagrams, we observe the larger band gap for the unit cell optimized using the 
(a)

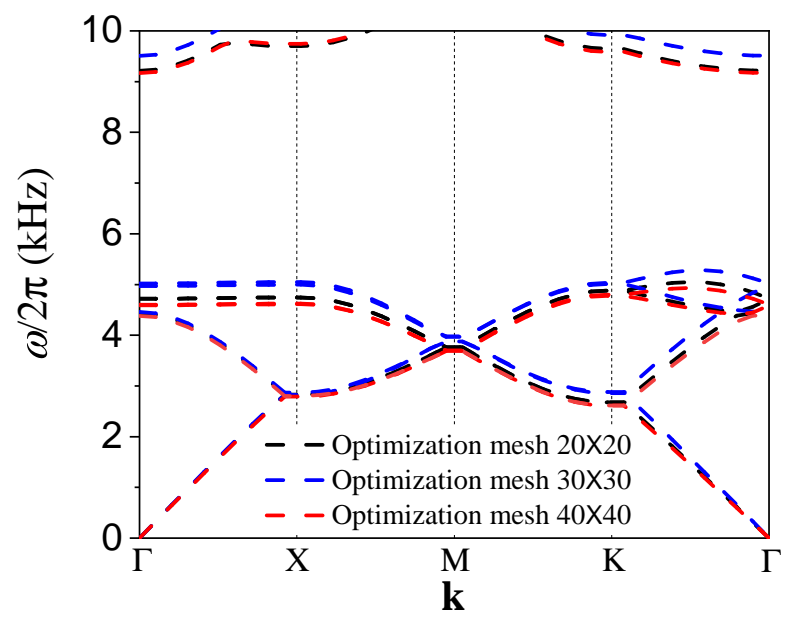

(b)

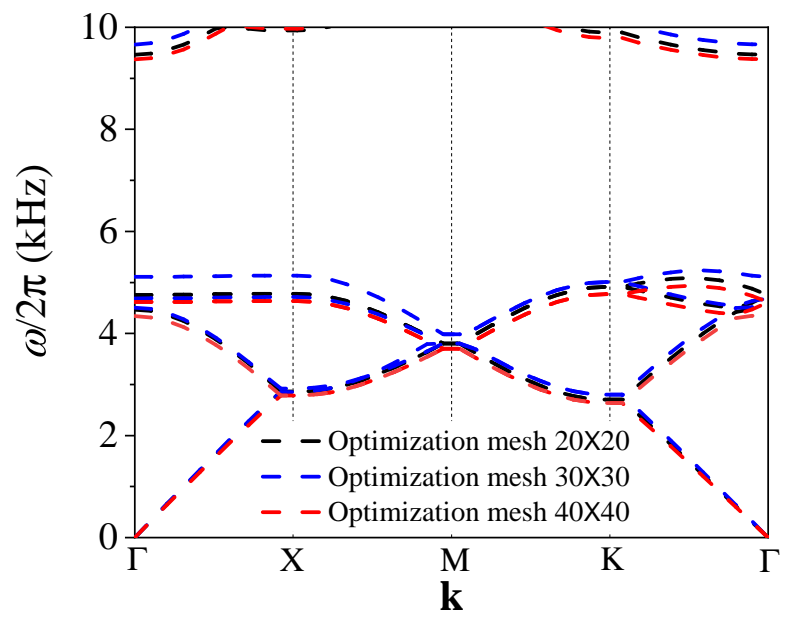

Figure 11: Comparison of the band diagrams for $120 \times 120$ mesh resolution of the unit cells optimized at $20 \times 20,30 \times 30$, and $40 \times 40$ mesh densities, (a) when bias electric field $e_{3}=150$, and (b) when bias electric field $e_{3}=300 \mathrm{MV} / \mathrm{m}$.

Table 5: Band gap between $4^{\text {th }}$ and $5^{\text {th }}$ bands at $120 \times 120$ mesh resolution for the unit cells optimized on $20 \times 20,30 \times 30$ and $40 \times 40$ mesh densities.

\begin{tabular}{|c|c|c|c|c|c|c|}
\hline \multirow{2}{*}{$\begin{array}{c}\text { Optimization mesh density } \rightarrow \\
\text { Bias electric field }\left(e_{3}\right) \rightarrow\end{array}$} & \multicolumn{2}{|c|}{$20 \times 20$} & \multicolumn{2}{|c|}{$30 \times 30$} & \multicolumn{2}{|c|}{$40 \times 40$} \\
\hline & $150 \mathrm{MV} / \mathrm{m}$ & $300 \mathrm{MV} / \mathrm{m}$ & $150 \mathrm{MV} / \mathrm{m}$ & $300 \mathrm{MV} / \mathrm{m}$ & $150 \mathrm{MV} / \mathrm{m}$ & $300 \mathrm{MV} / \mathrm{m}$ \\
\hline Optimized unit cells & & & & & & \\
\hline Band gap at $120 \times 120$ mesh resolution & $4.17 \mathrm{kHz}$ & $4.38 \mathrm{kHz}$ & $4.22 \mathrm{kHz}$ & $4.43 \mathrm{kHz}$ & $4.24 \mathrm{kHz}$ & $4.44 \mathrm{kHz}$ \\
\hline
\end{tabular}

gradient-based formulation $(4.37 \mathrm{kHz})$ in comparison to the one optimized using GA $(4.13 \mathrm{kHz})$. The present approach also holds a significant computational advantage: the computational cost of the GA is higher by several orders of magnitude, and this limits the achievable design resolution. For example, Bortot et al. (2018) reported that 12,500 function evaluations were required for GA to converge in a problem with 100 design variables. Herein, these problems are solved in less than 60 design cycles, where each cycle requires a single function evaluation and the computation of gradients. The cost of computing gradients can be estimated as roughly the same as a single function evaluation. As shown above, larger design freedom can yield better band gap performance, and this can only be achieved with the gradient-based approach that easily scales to finer mesh resolutions.

\section{Summary}

When subjected to electric fields, dielectric elastomers undergo finite deformations and their instantaneous moduli change. These features can be exploited to electrostatically tune the width of band gaps, across which waves cannot propagate in dielectric elastomer composites. 
(a)

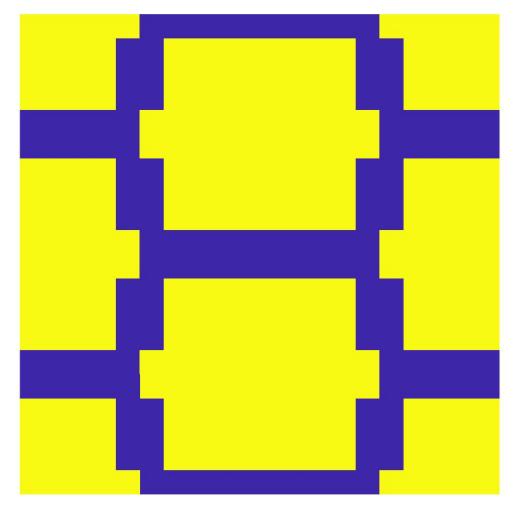

(b)

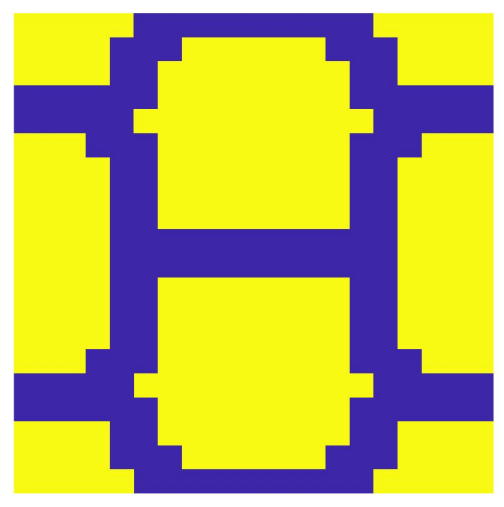

(c)

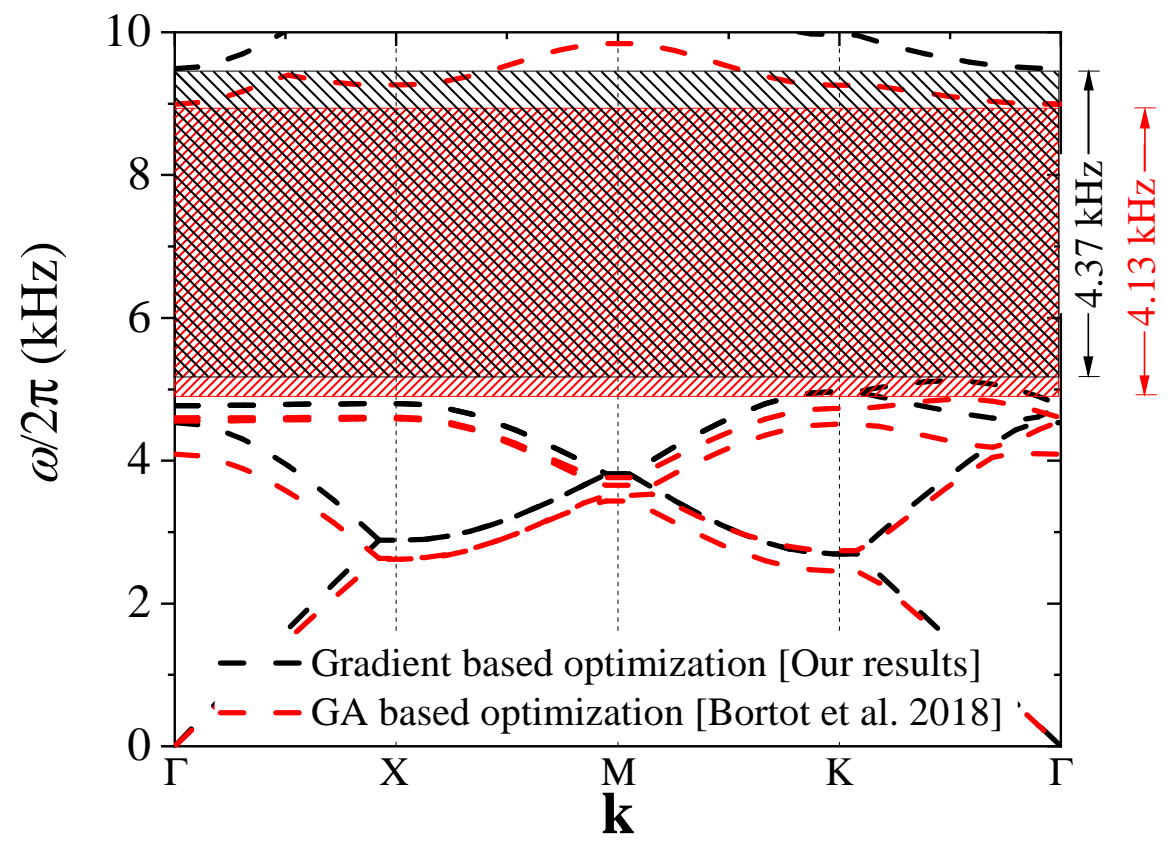

Figure 12: Designs of the unit cells optimized using (a) the gradient based optimization formulation presented in this paper and (b) a GA-based optimization method (Bortot et al., 2018). (c) Comparison of the corresponding band diagrams.

The width of these gaps in the actuated state is a function of the microstructure of the composite. To date, to identify optimal microstructures with wider gaps in the actuated state, topology optimization methods based on metaheuristics, specifically genetic algorithms, were used. These methods, which utilize only function evaluations without any gradient information, are limited by the computational cost that increases exponentially with the number of design variables.

In this work, we have developed a gradient-based topology optimization method, which utilizes information from the gradient of the objective function in order to iteratively update the design variables (Svanberg, 1987). The case study to which we have developed the method is of incremental anti-plane shear waves, propagating in a planar dielectric elastomer composite that is subjected to 
axial electric fields (Shmuel, 2013; Getz \& Shmuel, 2017; Bortot \& Shmuel, 2017). To this end, we have employed a finite element formulation to obtain the eigenmodes of the linearized problem, and extract the band diagram (Benchabane et al., 2006, Li et al., 2012; Veres et al., 2013). Based on finite element formulation, we have derived and implemented a fully analytical sensitivity analysis for computing the gradient of the objective function: the width of the gap in the audible range, at two prescribed electric fields.

In comparison with the genetic algorithm-based method (Bortot et al., 2018), our gradient-based method not only identified microstructures with wider gaps: it also reached these microstructures in an orders-of-magnitude cheaper computational cost. Thus, the gradient-based approach easily scales to finer mesh resolutions.

As a potential future work, the present topology optimization framework can extended to optimize band gaps associated with in-plane motions (Getz et al., 2017); and account for the viscosity of the elastomers by the integration of viscoelastic theory (Mohajer et al., 2021; Destrade \& Saccomandi, 2004; Chiang Foo et al., 2012, Hong, 2011).

\section{Acknowledgement}

The authors are thankful to the anonymous reviewers for their valuable input. This research was supported by the Israel Science Foundation, funded by the Israel Academy of Sciences and Humanities (Grant no. 2061/20), the United States-Israel Binational Science Foundation (Grant no. 2014358), and Ministry of Science and Technology (grant no. 880011).

\section{Appendix: Smooth formulation with approximate maximum and minimum eigenfrequencies along the wave vector $k$}

The objective function of the optimization problem in Eq. (36) is not strictly differentiable, because the $\mathrm{k}$-vector(s) corresponding to the maximum and minimum eigenfrequencies may change during the optimization iterations. Here, we present a topology optimization formulation in which the maximum and minimum values of eigenfrequencies along the wave vector $\mathbf{k}$ are approximated using a $p$-norm function, and compare the results for one test case to those in Section 5 . We examine the maximization of the band gap between the fourth and fifth bands which have a maximum optimized band gap in the audible frequency range.

Utilizing the standard $p$-norm measure (Duysinx \& Sigmund, 1998; Le et al., 2010), the maximum and minimum values of the eigenfrequencies corresponding to lower and upper bands, respectively, are approximated as

$$
\omega_{\max }^{P N}=\left(\sum_{i=1}^{n}\left(\omega_{i}\right)^{p}\right)^{\frac{1}{p}} ; \quad \omega_{\min }^{P N}=\frac{1}{\left(\sum_{i=1}^{n}\left(\frac{1}{\omega_{i}}\right)^{p}\right)^{\frac{1}{p}}},
$$

where, $p$ is the $p$-norm parameter, $\omega_{i}$ represents the value of the eigenfrequency corresponding to the $i^{\text {th }}$ point along the wave vector $\mathbf{k}$. In this work, we took $p=80$ and $n=40$ points along the wave vector $k$. 
(a)

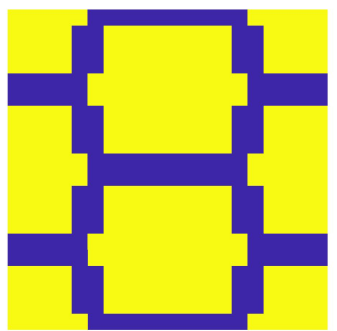

(b)

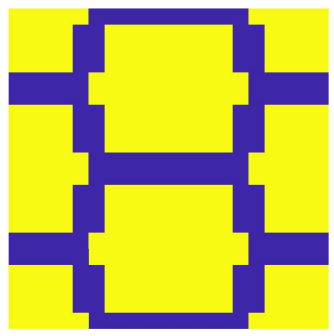

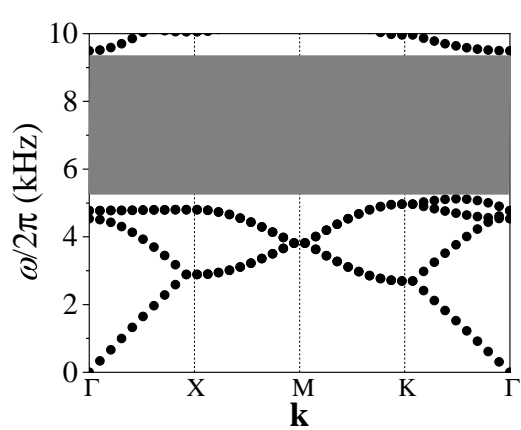
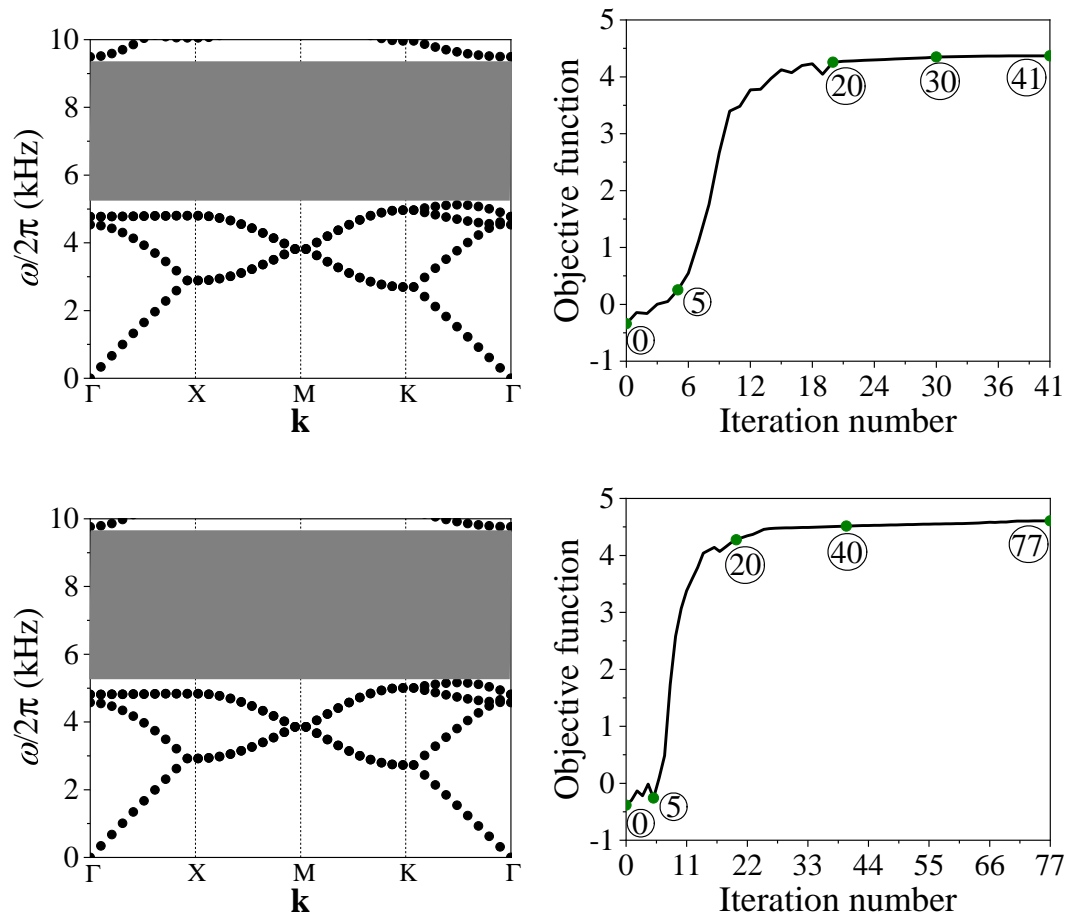

Figure 13: Unit cells (left), band diagrams (middle), and objective function history (right) when maximizing the band gap between $4^{\text {th }}$ and $5^{\text {th }}$ bands using the $p$-norm formulation, (a) when bias electric field $e_{3}=150 \mathrm{MV} / \mathrm{m}$, and (b) when bias electric field $e_{3}=300 \mathrm{MV} / \mathrm{m}$,

The modified version of the objective function is written as

$$
f^{P N}=\left\{\begin{array}{c}
10 \mathrm{kHz}-\omega_{\max }^{P N} / 2 \pi \\
\text { if } \min \omega_{j+1}(\xi, \mathbf{k}) / 2 \pi \geq 10 \mathrm{kHz} \\
\left(\omega_{\min }^{P N}-\omega_{\max }^{P N}\right) / 2 \pi \\
\text { if } \min \omega_{j+1}(\xi, \mathbf{k}) / 2 \pi \leq 10 \mathrm{kHz} .
\end{array}\right.
$$

and the sensitivities of the maximum and minimum frequencies approximated using $p$-norm with respect to design variable $\xi_{e}$ are expressed as

$$
\begin{aligned}
\frac{\partial \omega_{\max }^{P N}}{\partial \xi_{e}} & =\left(\sum_{i=1}^{n}\left(\omega_{i}\right)^{p}\right)^{\frac{1}{p}-1}\left(\sum_{i=1}^{n}\left(\omega_{i}\right)^{p-1} \frac{\partial \omega_{i}}{\partial \xi_{e}}\right) \\
\frac{\partial \omega_{\min }^{P N}}{\partial \xi_{e}} & =\left(\sum_{i=1}^{n}\left(\frac{1}{\omega_{i}}\right)^{p}\right)^{-1-\frac{1}{p}}\left(\sum_{i=1}^{n}\left(\frac{1}{\omega_{i}}\right)^{p+1} \frac{\partial \omega_{i}}{\partial \xi_{e}}\right)
\end{aligned}
$$

453 in which the derivative $\frac{\partial \omega_{i}}{\partial \xi_{e}}$ is evaluated using Eq. (39). Further, utilizing Eq. (A.3), we evaluated 454 the gradient of the modified version of the objective function $\frac{\partial f^{P N}}{\partial \xi_{e}}$ used for updating the design variables. 
(a)

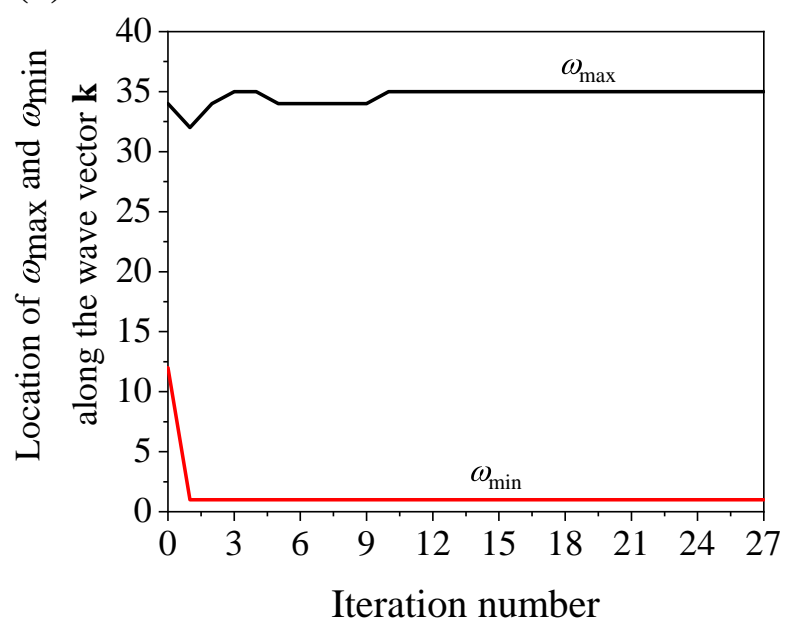

(b)

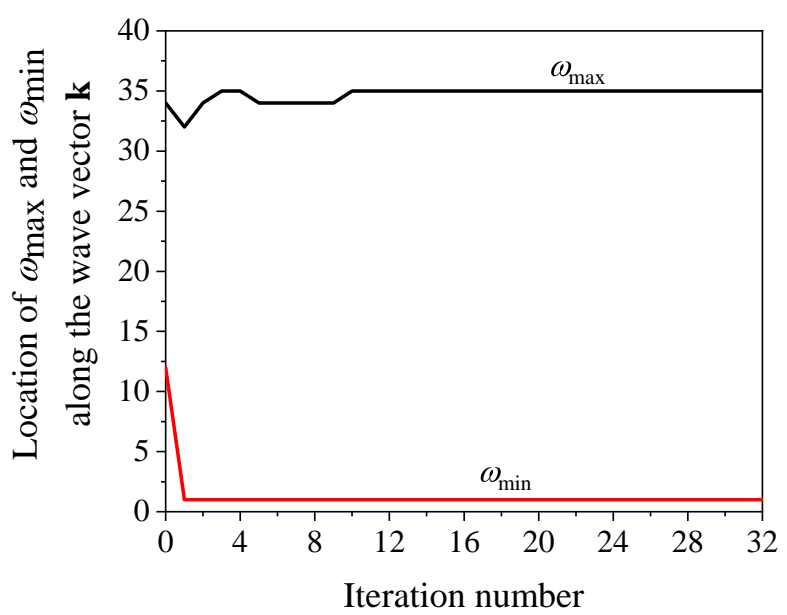

Figure 14: Variation of the location of the $\omega_{\max }$ and $\omega_{\min }$ along the wave vector $\mathbf{k}$, for the optimzation of the band gap between $4^{\text {th }}$ and $5^{\text {th }}$ bands using discrete formulation (Section 5), at (a) bias electric field $e_{3}=150 \mathrm{MV} / \mathrm{m}$ and (c) bias electric field $e_{3}=300 \mathrm{MV} / \mathrm{m}$.

The optimized layouts of the unit cell, the corresponding band diagrams, and objective function history obtained using the aforementioned $p$-norm formulation, for the maximization of the band gap between the $4^{\text {th }}$ and $5^{\text {th }}$ bands are shown in Figs. 13 and $13 \mathrm{~b}$, when bias electric field is equal to $e_{3}=150 \mathrm{MV} / \mathrm{m}$ and $e_{3}=300 \mathrm{MV} / \mathrm{m}$, respectively. From the optimized unit cell designs, we observe that the designs are the same as those obtained using the original formulation (Figs. $8 \mathrm{c}$ and 9c) for both values of bias electric field. In Fig. 114, we show that the location of $\omega_{\max }$ and $\omega_{\min }$ along the wave vector $\mathrm{k}$ is almost constant same for all iterations of the non-smooth formulation. This explains why the same designs are obtained using both formulations. However, from the right panel of Figs. $13 \mathrm{a}$ and $13 \mathrm{~b}$, we observe that the optimization with $p$-norms takes 41 (for $e_{3}=150 \mathrm{MV} / \mathrm{m}$ ) and 77 (for $e_{3}=300 \mathrm{MV} / \mathrm{m}$ ) iterations to converge, which consumes 14 (for $e_{3}=150 \mathrm{MV} / \mathrm{m}$ ) and 39 (for $e_{3}=300 \mathrm{MV} / \mathrm{m}$ ) more iterations in comparison to the non-smooth formulation (Figs. 88 and 9c). Further, the computational cost of optimization with the $p$-norm formulation is significantly higher in comparison to the non-smooth formulation, due to the evaluation of design sensitivities or the derivatives of eigenvalues corresponding to different values of wave-vector $\mathbf{k}$ along the first Brillouin zone.

\section{References}

Benchabane, S., Khelif, A., Rauch, J.-Y., Robert, L., \& Laude, V. (2006). Evidence for complete surface wave band gap in a piezoelectric phononic crystal. Physical Review E, 73, 065601.

Bendsøe, M., \& Kikuchi, N. (1988). Generating optimal topologies in structural design using a homogenization method. Computer Methods in Applied Mechanics and Engineering, 71, 197 - 224. URL: http://www.sciencedirect.com/science/article/pii/ 0045782588900862 , doi:https://doi.org/10.1016/0045-7825(88)90086-2.

Bilal, O., \& Hussein, M. (2011). Ultrawide phononic band gap for combined in-plane and out-ofplane waves. Physical Review E, 84, 065701. doi:10.1103/PhysRevE.84.065701. 
Bortot, E., Amir, O., \& Shmuel, G. (2018). Topology optimization of dielectric elastomers for wide tunable band gaps. International Journal of Solids and Structures, 143, 262-73.

Bortot, E., \& Shmuel, G. (2017). Tuning sound with soft dielectrics. Smart Materials and Structures, 26, 045028.

Chen, Y., Meng, F., Li, G., \& Huang, X. (2018). Designing photonic materials with complete band gaps by topology optimization. Smart Materials and Structures, 28, 015025.

Chiang Foo, C., Cai, S., Jin Adrian Koh, S., Bauer, S., \& Suo, Z. (2012). Model of dissipative dielectric elastomers. Journal of Applied Physics, 111, 034102.

De Pascalis, R., Donateo, T., Ficarella, A., \& Parnell, W. J. (2020). Optimal design of phononic media through genetic algorithm-informed pre-stress for the control of antiplane wave propagation. Extreme Mechanics Letters, 40, 100896.

Deaton, J. D., \& Grandhi, R. V. (2014). A survey of structural and multidisciplinary continuum topology optimization: post 2000. Structural and Multidisciplinary Optimization, 49, 1-38.

DeBotton, G., Tevet-Deree, L., \& Socolsky, E. A. (2007). Electroactive heterogeneous polymers: analysis and applications to laminated composites. Mech. Adv. Mater. Struct., 14, 13-22.

Destrade, M., \& Saccomandi, G. (2004). Finite-amplitude inhomogeneous waves in mooney-rivlin viscoelastic solids. Wave Motion, 40, 251-62.

Dorfmann, A., \& Ogden, R. (2005). Nonlinear electroelasticity. Acta Mechanica, 174, 167-83.

Dorfmann, A., \& Ogden, R. W. (2010). Electroelastic waves in a finitely deformed electroactive material. IMA Journal of Applied Mathematics, 75, 603-36.

Duysinx, P., \& Sigmund, O. (1998). New developments in handling stress constraints in optimal material distribution. In 7th AIAA/USAF/NASA/ISSMO symposium on multidisciplinary analysis and optimization (p. 4906).

Felippa, C. (2001). Introduction to finite element methods. multifreedom constraints ii (lecture notes).

Gazonas, G., Weile, D., Wildman, R., \& Mohan, A. (2006). Genetic algorithm optimization of phononic bandgap structures. International Journal of Solids and Structures, 43, 5851-66. doi:10.1016/j.ijsolstr.2005.12.002.

Gei, M., Roccabianca, S., \& Bacca, M. (2011). Controlling bandgap in electroactive polymer-based structures. IEEE-ASME Trans. Mechatronics, 16, 102-7.

Gei, M., Springhetti, R., \& Bortot, E. (2013). Performance of soft dielectric laminated composites. Smart Materials and Structures, 22, 104014. URL: https://doi .org/10. 1088/0964-1726/22/10/104014, doi:10.1088/0964-1726/22/10/104014.

Getz, R., Kochmann, D. M., \& Shmuel, G. (2017). Voltage-controlled complete stopbands in two-dimensional soft dielectrics. International Journal of Solids and Structures, 113, 24-36. 
Getz, R., \& Shmuel, G. (2017). Band gap tunability in deformable dielectric composite plates. International Journal of Solids and Structures, 128, 11-22.

Gu, G., Zou, J., Zhao, R., Zhao, X., \& Zhu, X. (2018). Soft wall-climbing robots. Science Robotics, 3.

Hajiesmaili, E., \& Clarke, D. R. (2021). Dielectric elastomer actuators. Journal of Applied Physics, 129, 151102.

Halkjær, O., S.and Sigmund, \& Jensen, J. (2006). Maximizing band gaps in plate structures. Structural and Multidisciplinary Optimization, 32, 263-75. doi:https://doi.org/10. 1007/s00158-006-0037-7.

Hedayatrasa, S., Abhary, K., Uddin, M., \& Guest, J. (2016). Optimal design of tunable phononic bandgap plates under equibiaxial stretch. Smart Materials and Structures, 25, 055025. doi:http://dx.doi.org/10.1088/0964-1726/25/5/055025.

Hong, W. (2011). Modeling viscoelastic dielectrics. Journal of the Mechanics and Physics of Solids, 59, 637-50.

Huang, K.-X., Shui, G.-S., Wang, Y.-Z., \& Wang, Y.-S. (2020). Meta-arrest of a fast propagating crack in elastic wave metamaterials with local resonators. Mechanics of Materials, 148, 103497.

Huang, K.-X., Shui, G.-S., Wang, Y.-Z., \& Wang, Y.-S. (2021). Enhanced fracture resistance induced by coupling multiple degrees of freedom in elastic wave metamaterials with local resonators. Journal of Elasticity, 144, 33-53.

Hussein, M., Hamza, K., Hulbert, G., \& Saitou, K. (2007). Optimal synthesis of 2d phononic crystals for broadband frequency isolation. Waves in Random and Complex Media, 17, 491-510. doi: $10.1080 / 17455030701501869$

Jandron, M., \& Henann, D. L. (2018). A numerical simulation capability for electroelastic wave propagation in dielectric elastomer composites: Application to tunable soft phononic crystals. International Journal of Solids and Structures, 150, 1-21.

Kashyap, K., Sharma, A. K., \& Joglekar, M. M. (2020). Nonlinear dynamic analysis of anisovisco-hyperelastic dielectric elastomer actuators. Smart Materials and Structures, 29, 055014.

Khurana, A., Kumar, D., Sharma, A. K., \& Joglekar, M. M. (2021). Nonlinear oscillations of particle-reinforced electro-magneto-viscoelastomer actuators. Journal of Applied Mechanics, 88,121002 .

Kim, C.-C., Lee, H.-H., Oh, K. H., \& Sun, J.-Y. (2016). Highly stretchable, transparent ionic touch panel. Science, 353, 682-7.

Kittel, C., McEuen, P., \& McEuen, P. (1996). Introduction to solid state physics volume 8. Wiley New York.

Kornbluh, R., \& Pelrine, R. (2008). Dielectric Elastomers as Electromechanical Transducers. chapter High-perfo. (pp. 33-42). Elsevier, Oxford, UK. 
Kushwaha, M. S., Halevi, P., Dobrzynski, L., \& Djafari-Rouhani, B. (1993). Acoustic band structure of periodic elastic composites. Physical review letters, 71, 2022.

Kushwaha, M. S., Halevi, P., Martinez, G., Dobrzynski, L., \& Djafari-Rouhani, B. (1994). Theory of acoustic band structure of periodic elastic composites. Physical Review B, 49, 2313.

Le, C., Norato, J., Bruns, T., Ha, C., \& Tortorelli, D. (2010). Stress-based topology optimization for continua. Structural and Multidisciplinary Optimization, 41, 605-20.

Li, J.-B., Wang, Y.-S., \& Zhang, C. (2012). Dispersion relations of a periodic array of fluid-filled holes embedded in an elastic solid. Journal of Computational Acoustics, 20, 1250014.

Li, W., Meng, F., Chen, Y., Li, Y. f., \& Huang, X. (2019). Topology optimization of photonic and phononic crystals and metamaterials: a review. Advanced Theory and Simulations, 2, 1900017.

Li, Y., Huang, X., Meng, F., \& Zhou, S. (2016a). Evolutionary topological design for phononic band gap crystals. Structural and Multidisciplinary Optimization, 54, 595-617. doi:10 . $1007 /$ s00158-016-1424-3.

Li, Y. f., Huang, X., Meng, F., \& Zhou, S. (2016b). Evolutionary topological design for phononic band gap crystals. Structural and Multidisciplinary Optimization, 54, 595-617.

Liu, W., Yoon, G. H., Yi, B., Choi, H., \& Yang, Y. (2020). Controlling wave propagation in onedimensional structures through topology optimization. Computers \& Structures, 241, 106368.

Liu, Z., Wu, B., \& He, C. (2014). Band-gap optimization of two-dimensional phononic crystals based on genetic algorithm and FPWE. Waves in Random and Complex Media, 24, 286-305. doi:10.1080/17455030.2014.901582.

Liu, Z., Wu, B., \& He, C. (2016a). Systematic topology optimization of solid-solid phononic crystals for multiple separate band-gaps with different polarizations. Ultrasonics, 65, 249-57. doi:10.1016/j.ultras.2015.09.017.

Liu, Z.-F., Wu, B., \& He, C.-F. (2016b). The properties of optimal two-dimensional phononic crystals with different material contrasts. Smart Materials and Structures, 25, 095036.

Lu, T., Shi, Z., Shi, Q., \& Wang, T. (2016). Bioinspired bicipital muscle with fiber-constrained dielectric elastomer actuator. Extreme Mechanics Letters, 6, 75-81.

Lu, Y., Yang, Y., Guest, J., \& Srivastava, A. (2017). 3-D phononic crystals with ultra-wide band gaps. Scientific Reports, 7, 43407 EP -. URL: http://dx.doi.org/10.1038/ srep 43407

Lustig, B., \& Shmuel, G. (2018). On the band gap universality of multiphase laminates and its applications. Journal of the Mechanics and Physics of Solids, 117, 37-53. URL: http://www.sciencedirect.com/science/article/pii/ S0022509618302321. doi:https://doi.org/10.1016/j.jmps.2018.04.008

Meng, F., Li, Y., Li, S., Lin, H., Jia, B., \& Huang, X. (2017). Achieving large band gaps in 2d symmetric and asymmetric photonic crystals. Journal of Lightwave Technology, 35, 1670-6. 
Mohajer, M., Zhou, J., \& Jiang, L. (2021). Small amplitude rayleigh-lamb wave propagation in a finitely deformed viscoelastic dielectric elastomer (de) layer. International Journal of Solids and Structures, 208-209, 93-106. URL: https://www.sciencedirect. com/science/article/pii/s0020768320303917, doi:https://doi.org/10. $1016 / j . i j s o l s t r .2020 .10 .006$.

Oliveira, J., Pinho-da Cruz, J., Andrade-Campos, A., \& Teixeira-Dias, F. (2010). Stress-and strainbased multifreedom constraints for periodic media optimisation. Actas da EngOpt, .

Ortigosa, R., \& Martínez-Frutos, J. (2021). Multi-resolution methods for the topology optimization of nonlinear electro-active polymers at large strains. Computational Mechanics, (pp. 1-23).

Ortigosa, R., Martínez-Frutos, J., Ruiz, D., Donoso, A., \& Bellido, J. (2021). Density-based topology optimisation considering nonlinear electromechanics. Structural and Multidisciplinary Optimization, (pp. 1-24).

Pelrine, R., Kornbluh, R., Pei, Q., \& Joseph, J. (2000). High-speed electrically actuated elastomers with strain greater than $100 \%$. Science, 287, 836-9.

Qian, X., \& Sigmund, O. (2011). Isogeometric shape optimization of photonic crystals via coons patches. Computer Methods in Applied Mechanics and Engineering, 200, 2237-55.

Quinteros, L., Meruane, V., \& Cardoso, E. L. (2021). Phononic band gap optimization in trusslike cellular structures using smooth p-norm approximations. Structural and Multidisciplinary Optimization, (pp. 1-12).

Ren, T., Li, F., Chen, Y., Liu, C., \& Zhang, C. (2020). Improvement of the band-gap characteristics of active composite laminate metamaterial plates. Composite Structures, 254, 112831.

Sharma, A. K. (2020). Design of a command-shaping scheme for mitigating residual vibrations in dielectric elastomer actuators. Journal of Applied Mechanics, 87.

Sharma, A. K., Arora, N., \& Joglekar, M. M. (2018). Dc dynamic pull-in instability of a dielectric elastomer balloon: an energy-based approach. Proceedings of the Royal Society A: Mathematical, Physical and Engineering Sciences, 474, 20170900.

Sharma, A. K., Bajpayee, S., Joglekar, D. M., \& Joglekar, M. M. (2017). Dynamic instability of dielectric elastomer actuators subjected to unequal biaxial prestress. Smart Materials and Structures, 26, 115019.

Sharma, A. K., Kumar, P., Singh, A., Joglekar, D. M., \& Joglekar, M. M. (2019). Electromechanical instability of dielectric elastomer actuators with active and inactive electric regions. Journal of Applied Mechanics, 86.

Sharma, A. K., Sheshkar, N., \& Gupta, A. (2021). Static and dynamic stability of dielectric elastomer fiber composites. Materials Today: Proceedings, 44, 2043-7.

Shmuel, G. (2013). Electrostatically tunable band gaps in finitely extensible dielectric elastomer fiber composites. International Journal of Solids and Structures, 50, 680-6. 
Shmuel, G., \& Band, R. (2016). Universality of the frequency spectrum of laminates. J. Mech. Phys. Solids, 92, 127-36. doi:http://dx.doi.org/10.1016/j.jmps.2016.04.001.

Shmuel, G., \& Debotton, G. (2012). Band-gaps in electrostatically controlled dielectric laminates subjected to incremental shear motions. J. Mech. Phys. Solids, 60, 1970-81. doi:10.1016/j . jmps.2012.05.006.

Shmuel, G., Gei, M., \& DeBotton, G. (2012). The $\{\mathrm{R}\}$ ayleigh- $\{\mathrm{L}\}$ amb wave propagation in dielectric elastomer layers subjected to large deformations. Int. J. Non-Linear. Mech., 47, 30716.

Shmuel, G., \& Pernas-Salomón, R. (2016). Manipulating motions of elastomer films by electrostatically-controlled aperiodicity. Smart Materials and Structures, 25, 125012.

Sigmund, O., \& Maute, K. (2013). Topology optimization approaches. Structural and Multidisciplinary Optimization, 48, 1031-55.

Sigmund, O., \& Søndergaard Jensen, J. (2003). Systematic design of phononic band-gap materials and structures by topology optimization. Philosophical Transactions of the Royal Society of London. Series A: Mathematical, Physical and Engineering Sciences, 361, 1001-19.

Su, Y., Chen, W., \& Destrade, M. (2019). Tuning the pull-in instability of soft dielectric elastomers through loading protocols. International Journal of Non-Linear Mechanics, 113, 62-6. URL: https://www.sciencedirect.com/science/article/pii/ S0020746218306863. doi:https://doi.org/10.1016/j.ijnonlinmec.2019. 03.008 .

Su, Y., Wu, B., Chen, W., \& Lü, C. (2018). Optimizing parameters to achieve giant deformation of an incompressible dielectric elastomeric plate. Extreme Mechanics Letters, 22, 60-8. doi:https://doi.org/10.1016/j.eml.2018.05.004.

Suo, Z., Zhao, X., \& Greene, W. H. (2008). A nonlinear field theory of deformable dielectrics. Journal of the Mechanics and Physics of Solids, 56, 467-86.

Svanberg, K. (1987). The method of moving asymptotes-a new method for structural optimization. International journal for numerical methods in engineering, 24, 359-73.

Vatanabe, S. L., Paulino, G. H., \& Silva, E. C. (2014). Maximizing phononic band gaps in piezocomposite materials by means of topology optimization. The Journal of the Acoustical Society of America, 136, 494-501.

Veres, I. A., Berer, T., \& Matsuda, O. (2013). Complex band structures of two dimensional phononic crystals: Analysis by the finite element method. Journal of Applied Physics, 114, 083519.

Wang, Y.-F., Wang, Y.-Z., Wu, B., Chen, W., \& Wang, Y.-S. (2020). Tunable and active phononic crystals and metamaterials. Applied Mechanics Reviews, 72. 
Xie, L., Xia, B., Liu, J., Huang, G., \& Lei, J. (2017). An improved fast plane wave expansion method for topology optimization of phononic crystals. International Journal of Mechanical Science, 120, 171-81. doi:http://dx.doi.org/10.1016/j.ijmecsci.2016.11. 023 .

Yang, X., \& Kim, Y. Y. (2018). Topology optimization for the design of perfect mode-converting anisotropic elastic metamaterials. Composite Structures, 201, 161-77.

Yi, G., Shin, Y. C., Yoon, H., Jo, S.-H., \& Youn, B. D. (2019). Topology optimization for phononic band gap maximization considering a target driving frequency. JMST Advances, 1, 153-9.

Yi, G., \& Youn, B. D. (2016). A comprehensive survey on topology optimization of phononic crystals. Structural and Multidisciplinary Optimization, 54, 1315-44.

Zhang, G., \& Gao, X.-L. (2018). Elastic wave propagation in 3-d periodic composites: Band gaps incorporating microstructure effects. Composite Structures, 204, 920-32.

Zhang, X., Xing, J., Liu, P., Luo, Y., \& Kang, Z. (2021). Realization of full and directional band gap design by non-gradient topology optimization in acoustic metamaterials. Extreme Mechanics Letters, 42, 101126.

Zhao, X., Hong, W., \& Suo, Z. (2007). Electromechanical hysteresis and coexistent states in dielectric elastomers. Physical review B, 76, 134113.

Zhu, F., Wu, B., Destrade, M., \& Chen, W. (2020). Electrostatically tunable axisymmetric vibrations of soft electro-active tubes. Journal of Sound and Vibration, 483, 115467. URL: https://www.sciencedirect.com/science/article/pii/ s0022460X20302996. doi:https://doi.org/10.1016/j.jsv.2020.115467.

Ziser, Y., \& Shmuel, G. (2017). Experimental slowing of flexural waves in dielectric elastomer films by voltage. Mech. Res. Commun., 85, 64-8. URL: http://www. sciencedirect. com/science/article/pii/s0093641317302197, doi:https://doi.org/10. $1016 / j . m e c h r e s c o m .2017 .08 .005$. 